\title{
Structural variations of the first family of heterometallic uranyl carboxyphosphinate assemblies by synergy between carboxyphosphinate and imidazole ligands
}

\author{
Weiting Yang, ${ }^{\mathrm{a}}$ Dai Wu, ${ }^{\mathrm{a}}$ Chao Liu, ${ }^{\mathrm{a}}$ Qing-Jiang Pan*,b ${ }^{*}$, and Zhong-Ming Sun *,a \\ ${ }^{\text {a }}$ State Key Laboratory of Rare Earth Resource Utilization, Changchun Institute of Applied \\ Chemistry, Chinese Academy of Sciences, 5625 Renmin Street, Changchun, Jilin 130022, China. \\ ${ }^{\mathrm{b}}$ Key Laboratory of Functional Inorganic Material Chemistry of Education Ministry, School of \\ Chemistry and Materials Science, Heilongiiang University, Harbin 150080, China.
}

\section{SUPPORTING INFORMATION}

\section{Tables.}

Table S1 Selected bond lengths and angles for 1-8.

Table S2 Average bond lengths of six-fold and seven-fold coordinated uranium cations in 1-8.

Table S3 IR spectroscopic data for 1-8.

Table S4 Comparison of selected single-crystal XRD and IR spectroscopic data for 1-8 using Veal's empirical law.

\section{Powder X-ray diffraction patterns.}

Figure S1. PXRD pattern of $\mathrm{Co}(\mathrm{im})_{2}\left(\mathrm{UO}_{2}\right)_{3}(\mathrm{~L})_{4} \mathbf{1}$.

Figure S2. PXRD pattern of Zn(bpi) $\left(\mathrm{UO}_{2}\right)(\mathrm{L})_{2} \mathbf{2}$.

Figure S3. PXRD pattern of $\mathrm{Mn}(\mathrm{dib})\left(\mathrm{UO}_{2}\right)_{2}(\mathrm{~L})_{3} \mathbf{5}$.

Figure S4. PXRD pattern of $\left[\mathrm{Co}(\mathrm{dib})_{2}\left(\mathrm{H}_{2} \mathrm{O}\right)_{2}\right]\left[\left(\mathrm{UO}_{2}\right)_{3}(\mathrm{~L})_{4}\right] \cdot 2 \mathrm{H}_{2} \mathrm{O} 6$.

Figure S5. PXRD pattern of $\left[\mathrm{Ni}(\mathrm{dib})_{2}\left(\mathrm{H}_{2} \mathrm{O}\right)_{2}\right]\left[\left(\mathrm{UO}_{2}\right)_{3}(\mathrm{~L})_{4}\right] \cdot 2 \mathrm{H}_{2} \mathrm{O} 7$.

\section{Infrared spectra}

Figure S6. IR spectrum of $\mathrm{Co}(\mathrm{im})_{2}\left(\mathrm{UO}_{2}\right)_{3}(\mathrm{~L})_{4} \mathbf{1}$.

Figure S7. IR spectrum of $\mathrm{Zn}(\mathrm{bpi})\left(\mathrm{UO}_{2}\right)(\mathrm{L})_{2} 2$.

Figure S8. IR spectrum of $\mathrm{Cd}(\mathrm{dib})\left(\mathrm{UO}_{2}\right)(\mathrm{L})_{2} 3$.

Figure S9. IR spectrum of $\mathrm{Cd}(\mathrm{dib})\left(\mathrm{UO}_{2}\right)_{2}(\mathrm{~L})_{3} 4$.

Figure S10. IR spectrum of $\mathrm{Mn}(\mathrm{dib})\left(\mathrm{UO}_{2}\right)_{2}(\mathrm{~L})_{3} 5$.

Figure S11. IR spectrum of $\left[\mathrm{Co}(\mathrm{dib})_{2}\left(\mathrm{H}_{2} \mathrm{O}\right)_{2}\right]\left[\left(\mathrm{UO}_{2}\right)_{3}(\mathrm{~L})_{4}\right] \cdot 2 \mathrm{H}_{2} \mathrm{O} 6$. 
Figure S12. IR spectrum of $\left[\mathrm{Ni}(\mathrm{dib})_{2}\left(\mathrm{H}_{2} \mathrm{O}\right)_{2}\right]\left[\left(\mathrm{UO}_{2}\right)_{3}(\mathrm{~L})_{4}\right] \cdot 2 \mathrm{H}_{2} \mathrm{O} 7$.

Figure S13. IR spectrum of $\left[\mathrm{Cu}(\mathrm{dib})_{2}\left(\mathrm{H}_{2} \mathrm{O}\right)_{2}\right]\left[\left(\mathrm{UO}_{2}\right)_{3}(\mathrm{~L})_{4}\right] \mathbf{8}$.

\section{ORTEP representation of local structures.}

Figure S14. ORTEP representation of local structure of Co(im $)_{2}\left(\mathrm{UO}_{2}\right)_{3}(\mathrm{~L})_{4} \mathbf{1}$.

Figure S15. ORTEP representation of local structure of $\mathrm{Zn}(\mathrm{bpi})\left(\mathrm{UO}_{2}\right)(\mathrm{L})_{2} 2$.

Figure S16. ORTEP representation of local structure of Cd(dib)(UO $\left.)_{2}\right)(\mathrm{L})_{2} 3$.

Figure S17. ORTEP representation of local structure of $\mathrm{Mn}(\mathrm{dib})\left(\mathrm{UO}_{2}\right)_{2}(\mathrm{~L})_{3} 5$.

Figure S18. ORTEP representation of local structure of $\left[\mathrm{Co}(\mathrm{dib})_{2}\left(\mathrm{H}_{2} \mathrm{O}\right)_{2}\right]\left[\left(\mathrm{UO}_{2}\right)_{3}(\mathrm{~L})_{4}\right] \cdot 2 \mathrm{H}_{2} \mathrm{O} 6$.

\section{V.Excitation and Emission spectra.}

Figure S19. Excitation and emission spectra of Co(im $)_{2}\left(\mathrm{UO}_{2}\right)_{3}(\mathrm{~L})_{4} \mathbf{1}$.

Figure S20. Excitation and emission spectra of $\mathrm{Mn}(\mathrm{dib})\left(\mathrm{UO}_{2}\right)_{2}(\mathrm{~L})_{3} \mathbf{5}$.

Figure S21. Excitation and emission spectra of $\left[\mathrm{Co}(\mathrm{dib})_{2}\left(\mathrm{H}_{2} \mathrm{O}\right)_{2}\right]\left[\left(\mathrm{UO}_{2}\right)_{3}(\mathrm{~L})_{4}\right] \cdot 2 \mathrm{H}_{2} \mathrm{O} 6$.

Figure S22. Excitation and emission spectra of $\left[\mathrm{Ni}(\mathrm{dib})_{2}\left(\mathrm{H}_{2} \mathrm{O}\right)_{2}\right]\left[\left(\mathrm{UO}_{2}\right)_{3}(\mathrm{~L})_{4}\right] \cdot 2 \mathrm{H}_{2} \mathrm{O} 7$.

Figure S23. Excitation and emission spectra of the carboxphosphinate ligand CPP.

Figure S24. Excitation and emission spectra of imidazole.

Figure S25. Excitation and emission spectra of bpi.

Figure S26. Excitation and emission spectra of dib. 


\section{Tables.}

Table S1 Selected bond lengths $(\AA)$ and angles $\left({ }^{\circ}\right)$ for $\mathbf{1 - 8}$.

\begin{tabular}{llll}
\hline $\mathbf{1}$ & & & \\
$\mathrm{U}(1)-\mathrm{O}(1) \# 1$ & $1.729(9)$ & $\mathrm{U}(2)-\mathrm{O}(3)$ & $1.695(9)$ \\
$\mathrm{U}(1)-\mathrm{O}(1)$ & $1.729(9)$ & $\mathrm{U}(2)-\mathrm{O}(2)$ & $1.733(8)$ \\
$\mathrm{U}(1)-\mathrm{O}(5)$ & $2.283(9)$ & $\mathrm{U}(2)-\mathrm{O}(11) \# 3$ & $2.290(8)$ \\
$\mathrm{U}(1)-\mathrm{O}(5) \# 1$ & $2.283(9)$ & $\mathrm{U}(2)-\mathrm{O}(4)$ & $2.301(9)$ \\
$\mathrm{U}(1)-\mathrm{O}(10) \# 2$ & $2.299(8)$ & $\mathrm{U}(2)-\mathrm{O}(6) \# 4$ & $2.305(9)$ \\
$\mathrm{U}(1)-\mathrm{O}(10) \# 3$ & $2.299(8)$ & $\mathrm{U}(2)-\mathrm{O}(9)$ & $2.465(9)$ \\
& & $\mathrm{U}(2)-\mathrm{O}(8)$ & $2.549(9)$ \\
$\mathrm{Co}(1)-\mathrm{O}(7) \# 4$ & $2.078(8)$ & $\mathrm{P}(1)-\mathrm{O}(4)$ & $1.507(9)$ \\
$\mathrm{Co}(1)-\mathrm{O}(7) \# 5$ & $2.078(8)$ & $\mathrm{P}(1)-\mathrm{O}(5)$ & $1.516(9)$ \\
$\mathrm{Co}(1)-\mathrm{N}(1) \# 6$ & $2.124(12)$ & $\mathrm{P}(1)-\mathrm{C}(7)$ & $1.759(13)$ \\
$\mathrm{Co}(1)-\mathrm{N}(1)$ & $2.124(12)$ & $\mathrm{P}(1)-\mathrm{C}(1)$ & $1.779(14)$ \\
& & $\mathrm{P}(2)-\mathrm{O}(11)$ & $1.504(9)$ \\
& & $\mathrm{P}(2)-\mathrm{O}(10)$ & $1.517(9)$ \\
$\mathrm{O}(1) \# 1-\mathrm{U}(1)-\mathrm{O}(1)$ & $179.998(1)$ & $\mathrm{P}(2)-\mathrm{C}(10)$ & $1.766(16)$ \\
$\mathrm{O}(3)-\mathrm{U}(2)-\mathrm{O}(2)$ & $175.3(4)$ & $\mathrm{P}(2)-\mathrm{C}(16)$ & $1.768(13)$
\end{tabular}

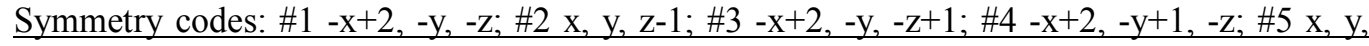
$\mathrm{z}+1 ; \# 6-\mathrm{x}+2,-\mathrm{y}+1,-\mathrm{z}+1$.

2

$\begin{array}{ll}\mathrm{U}(1)-\mathrm{O}(1) & 1.753(4) \\ \mathrm{U}(1)-\mathrm{O}(2) & 1.753(4) \\ \mathrm{U}(1)-\mathrm{O}(5) & 2.299(3) \\ \mathrm{U}(1)-\mathrm{O}(6) & 2.337(3) \\ \mathrm{U}(1)-\mathrm{O}(7) & 2.400(3) \\ \mathrm{U}(1)-\mathrm{O}(4) & 2.443(3) \\ \mathrm{U}(1)-\mathrm{O}(3) & 2.498(3) \\ \mathrm{Zn}(1)-\mathrm{O}(8) & 1.925(3) \\ \mathrm{Zn}(1)-\mathrm{O}(9) & 1.935(3) \\ \mathrm{Zn}(1)-\mathrm{O}(10) \# 1 & 1.951(3) \\ \mathrm{Zn}(1)-\mathrm{N}(1) & 1.974(4)\end{array}$

$\mathrm{P}(1)-\mathrm{O}(8)$

$\mathrm{P}(1)-\mathrm{O}(6)$

$\mathrm{P}(1)-\mathrm{C}(3)$

$\mathrm{P}(1)-\mathrm{C}(4)$

$\mathrm{P}(2)-\mathrm{O}(5)$

$\mathrm{P}(2)-\mathrm{O}(9)$

$\mathrm{P}(2)-\mathrm{C}(12)$

$\mathrm{P}(2)-\mathrm{C}(13)$

$\mathrm{O}(1)-\mathrm{U}(1)-\mathrm{O}(2)$
3

$\begin{array}{ll}\mathrm{U}(1)-\mathrm{O}(2) & 1.763(7) \\ \mathrm{U}(1)-\mathrm{O}(1) & 1.777(7) \\ \mathrm{U}(1)-\mathrm{O}(4) & 2.276(6) \\ \mathrm{U}(1)-\mathrm{O}(10) \# 1 & 2.307(7) \\ \mathrm{U}(1)-\mathrm{O}(8) \# 2 & 2.312(6) \\ \mathrm{U}(1)-\mathrm{O}(6) \# 1 & 2.464(7) \\ \mathrm{U}(1)-\mathrm{O}(5) \# 1 & 2.526(6) \\ \mathrm{Cd}(1)-\mathrm{O}(7) \# 3 & 2.228(7) \\ \mathrm{Cd}(1)-\mathrm{N}(3) \# 4 & 2.238(8) \\ \mathrm{Cd}(1)-\mathrm{O}(3) \# 5 & 2.266(6) \\ \mathrm{Cd}(1)-\mathrm{N}(1) & 2.290(8) \\ \mathrm{Cd}(1)-\mathrm{O}(9) & 2.384(7) \\ \mathrm{Cd}(1)-\mathrm{O}(5) & 2.512(7) \\ \mathrm{P}(1)-\mathrm{O}(3) & 1.482(7) \\ \mathrm{P}(1)-\mathrm{O}(4) & 1.523(7) \\ \mathrm{P}(1)-\mathrm{C}(1) & 1.779(12) \\ \mathrm{P}(1)-\mathrm{C}(7) & 1.786(10) \\ \mathrm{P}(2)-\mathrm{O}(7) & 1.483(7) \\ \mathrm{P}(2)-\mathrm{O}(8) & 1.519(7) \\ \mathrm{P}(2)-\mathrm{C}(16) & 1.787(10) \\ \mathrm{P}(2)-\mathrm{C}(10) & 1.793(13) \\ \mathrm{O}(2)-\mathrm{U}(1)-\mathrm{O}(1) & 178.3(3)\end{array}$


Symmetry codes: \#1 x, y+1, z for 2 ; \#1 -x+1, -y, $-\mathrm{z}+1$; \#2 $\mathrm{x}, \mathrm{y}, \mathrm{z}-1 ; \# 3-\mathrm{x},-\mathrm{y},-\mathrm{z}+2$; \#4 $\mathrm{x}+1$, $\mathrm{y}-1, \mathrm{z} ; \# 5-\mathrm{x},-\mathrm{y},-\mathrm{z}+1$ for 3 .

\begin{tabular}{|c|c|c|c|}
\hline \multicolumn{2}{|l|}{4} & \multicolumn{2}{|l|}{5} \\
\hline $\mathrm{U}(1)-\mathrm{O}(2)$ & $1.760(5)$ & $\mathrm{U}(1)-\mathrm{O}(1)$ & $1.742(7)$ \\
\hline $\mathrm{U}(1)-\mathrm{O}(1)$ & $1.769(5)$ & $\mathrm{U}(1)-\mathrm{O}(2)$ & $1.743(7)$ \\
\hline $\mathrm{U}(1)-\mathrm{O}(13) \# 1$ & $2.259(4)$ & $\mathrm{U}(1)-\mathrm{O}(13) \# 1$ & $2.277(6)$ \\
\hline $\mathrm{U}(1)-\mathrm{O}(3)$ & $2.275(5)$ & $\mathrm{U}(1)-\mathrm{O}(3)$ & $2.274(6)$ \\
\hline $\mathrm{U}(1)-\mathrm{O}(4)$ & $2.300(4)$ & $\mathrm{U}(1)-\mathrm{O}(4)$ & $2.277(6)$ \\
\hline $\mathrm{U}(1)-\mathrm{O}(5)$ & $2.277(5)$ & $\mathrm{U}(1)-\mathrm{O}(5)$ & $2.284(6)$ \\
\hline $\mathrm{U}(2)-\mathrm{O}(7)$ & $1.747(5)$ & $\mathrm{U}(2)-\mathrm{O}(7)$ & $1.744(8)$ \\
\hline $\mathrm{U}(2)-\mathrm{O}(8)$ & $1.750(5)$ & $\mathrm{U}(2)-\mathrm{O}(8)$ & $1.749(7)$ \\
\hline $\mathrm{U}(2)-\mathrm{O}(11) \# 1$ & $2.296(5)$ & $\mathrm{U}(2)-\mathrm{O}(11) \# 1$ & $2.266(7)$ \\
\hline $\mathrm{U}(2)-\mathrm{O}(9)$ & $2.313(4)$ & $\mathrm{U}(2)-\mathrm{O}(9)$ & $2.310(6)$ \\
\hline $\mathrm{U}(2)-\mathrm{O}(6) \# 1$ & $2.329(5)$ & $\mathrm{U}(2)-\mathrm{O}(6) \# 1$ & $2.326(7)$ \\
\hline $\mathrm{U}(2)-\mathrm{O}(15) \# 2$ & $2.469(5)$ & $\mathrm{U}(2)-\mathrm{O}(15) \# 2$ & $2.454(8)$ \\
\hline $\mathrm{U}(2)-\mathrm{O}(16) \# 2$ & $2.482(5)$ & $\mathrm{U}(2)-\mathrm{O}(16) \# 2$ & $2.504(7)$ \\
\hline $\mathrm{Cd}(1)-\mathrm{O}(14)$ & $2.215(5)$ & $\mathrm{Mn}(1)-\mathrm{O}(14)$ & $2.078(7)$ \\
\hline $\mathrm{Cd}(1)-\mathrm{O}(10) \# 3$ & $2.293(5)$ & $\mathrm{Mn}(1)-\mathrm{O}(10) \# 3$ & $2.161(8)$ \\
\hline $\mathrm{Cd}(1)-\mathrm{O}(12)$ & $2.324(5)$ & $\mathrm{Mn}(1)-\mathrm{O}(12)$ & $2.180(7)$ \\
\hline $\mathrm{Cd}(1)-\mathrm{N}(3)$ & $2.245(6)$ & $\mathrm{Mn}(1)-\mathrm{N}(3)$ & $2.184(9)$ \\
\hline $\mathrm{Cd}(1)-\mathrm{N}(1)$ & $2.254(6)$ & $\operatorname{Mn}(1)-\mathrm{N}(1)$ & $2.212(9)$ \\
\hline $\mathrm{P}(1)-\mathrm{O}(5)$ & $1.510(5)$ & $\mathrm{P}(1)-\mathrm{O}(5)$ & $1.515(7)$ \\
\hline $\mathrm{P}(1)-\mathrm{O}(6)$ & $1.514(5)$ & $\mathrm{P}(1)-\mathrm{O}(6)$ & $1.513(7)$ \\
\hline$P(1)-C(1)$ & $1.813(8)$ & $\mathrm{P}(1)-\mathrm{C}(1)$ & $1.771(11)$ \\
\hline $\mathrm{P}(1)-\mathrm{C}(7)$ & $1.768(7)$ & $\mathrm{P}(1)-\mathrm{C}(7)$ & $1.794(9)$ \\
\hline $\mathrm{P}(2)-\mathrm{O}(14)$ & $1.475(5)$ & $\mathrm{P}(2)-\mathrm{O}(14)$ & $1.480(7)$ \\
\hline $\mathrm{P}(2)-\mathrm{O}(3)$ & $1.509(5)$ & $\mathrm{P}(2)-\mathrm{O}(3)$ & $1.522(6)$ \\
\hline$P(2)-C(10)$ & $1.787(8)$ & $\mathrm{P}(2)-\mathrm{C}(11)$ & $1.771(12)$ \\
\hline $\mathrm{P}(2)-\mathrm{C}(16)$ & $1.811(7)$ & $\mathrm{P}(2)-\mathrm{C}(17)$ & $1.835(15)$ \\
\hline $\mathrm{P}(3)-\mathrm{O}(9)$ & $1.493(5)$ & $\mathrm{P}(3)-\mathrm{O}(9)$ & $1.501(7)$ \\
\hline $\mathrm{P}(3)-\mathrm{O}(4)$ & $1.509(5)$ & $\mathrm{P}(3)-\mathrm{O}(4)$ & $1.528(7)$ \\
\hline$P(3)-C(25)$ & $1.792(7)$ & $\mathrm{P}(3)-\mathrm{C}(27)$ & $1.780(10)$ \\
\hline $\mathrm{P}(3)-\mathrm{C}(19)$ & $1.799(7)$ & $\mathrm{P}(3)-\mathrm{C}(21)$ & $1.783(11)$ \\
\hline $\mathrm{O}(2)-\mathrm{U}(1)-\mathrm{O}(1)$ & $178.2(2)$ & $\mathrm{O}(1)-\mathrm{U}(1)-\mathrm{O}(2)$ & $178.4(3)$ \\
\hline $\mathrm{O}(7)-\mathrm{U}(2)-\mathrm{O}(8)$ & $177.3(2)$ & $\mathrm{O}(7)-\mathrm{U}(2)-\mathrm{O}(8)$ & $177.0(4)$ \\
\hline \multicolumn{4}{|c|}{ Symmetry codes: \#1 x-1, y, z; \#2 x, y+1, z; \#3 x, y-1, z. } \\
\hline & $6(\mathrm{Co})$ & $7(\mathrm{Ni})$ & $8(\mathrm{Cu})$ \\
\hline $\mathrm{U}(1)-\mathrm{O}(1) \# 1$ & $1.774(6)$ & $1.769(5)$ & $1.775(5)$ \\
\hline $\mathrm{U}(1)-\mathrm{O}(1)$ & $1.774(6)$ & $1.769(5)$ & $1.775(5)$ \\
\hline $\mathrm{U}(1)-\mathrm{O}(11) \# 2$ & $2.256(6)$ & $2.262(4)$ & $2.258(4)$ \\
\hline $\mathrm{U}(1)-\mathrm{O}(11) \# 3$ & $2.256(6)$ & $2.262(4)$ & $2.258(4)$ \\
\hline $\mathrm{U}(1)-\mathrm{O}(4)$ & $2.279(5)$ & $2.285(4)$ & $2.288(5)$ \\
\hline $\mathrm{U}(1)-\mathrm{O}(4) \# 1$ & $2.279(5)$ & $2.285(4)$ & $2.288(5)$ \\
\hline $\mathrm{U}(2)-\mathrm{O}(2)$ & $1.769(6)$ & $1.762(5)$ & $1.764(5)$ \\
\hline
\end{tabular}




\begin{tabular}{lccc}
$\mathrm{U}(2)-\mathrm{O}(3)$ & $1.776(6)$ & $1.768(5)$ & $1.771(5)$ \\
$\mathrm{U}(2)-\mathrm{O}(8)$ & $2.299(6)$ & $2.296(4)$ & $2.305(4)$ \\
$\mathrm{U}(2)-\mathrm{O}(10) \# 4$ & $2.317(5)$ & $2.326(4)$ & $2.312(5)$ \\
$\mathrm{U}(2)-\mathrm{O}(5) \# 5$ & $2.322(6)$ & $2.327(4)$ & $2.335(4)$ \\
$\mathrm{U}(2)-\mathrm{O}(7)$ & $2.441(6)$ & $2.437(5)$ & $2.442(5)$ \\
$\mathrm{U}(2)-\mathrm{O}(6)$ & $2.507(5)$ & $2.506(4)$ & $2.507(5)$ \\
$\mathrm{M}(1)-\mathrm{N}(1)$ & $2.122(6)$ & $2.075(5)$ & $1.993(5)$ \\
$\mathrm{M}(1)-\mathrm{N}(1) \# 6$ & $2.122(6)$ & $2.075(5)$ & $1.993(5)$ \\
$\mathrm{M}(1)-\mathrm{N}(3)$ & $2.153(7)$ & $2.121(5)$ & $2.046(5)$ \\
$\mathrm{M}(1)-\mathrm{N}(3) \# 6$ & $2.153(7)$ & $2.121(5)$ & $2.046(5)$ \\
$\mathrm{M}(1)-\mathrm{O}(1 \mathrm{~W})$ & $2.137(6)$ & $2.116(5)$ & $2.502(6)$ \\
$\mathrm{M}(1)-\mathrm{O}(1 \mathrm{~W}) \# 6$ & $2.137(6)$ & $2.116(5)$ & $2.502(6)$ \\
$\mathrm{P}(1)-\mathrm{O}(5)$ & $1.505(6)$ & $1.505(4)$ & $1.503(5)$ \\
$\mathrm{P}(1)-\mathrm{O}(4)$ & $1.518(5)$ & $1.513(5)$ & $1.522(5)$ \\
$\mathrm{P}(1)-\mathrm{C}(7)$ & $1.781(8)$ & $1.780(7)$ & $1.782(8)$ \\
$\mathrm{P}(1)-\mathrm{C}(1)$ & $1.803(9)$ & $1.795(8)$ & $1.796(8)$ \\
$\mathrm{P}(2)-\mathrm{O}(10)$ & $1.516(5)$ & $1.508(4)$ & $1.511(5)$ \\
$\mathrm{P}(2)-\mathrm{O}(11)$ & $1.523(6)$ & $1.522(4)$ & $1.520(4)$ \\
$\mathrm{P}(2)-\mathrm{C}(16)$ & $1.777(9)$ & $1.782(7)$ & $1.783(8)$ \\
$\mathrm{P}(2)-\mathrm{C}(10)$ & $1.795(9)$ & $1.789(7)$ & $1.789(7)$ \\
$\mathrm{O}(1) \# 1-\mathrm{U}(1)-\mathrm{O}(1)$ & $180.000(1)$ & $180.000(1)$ & $180.0(6)$ \\
$\mathrm{O}(2)-\mathrm{U}(2)-\mathrm{O}(3)$ & $178.4(3)$ & $178.3(2)$ & $178.1(2)$ \\
$\mathrm{Symmetry} \mathrm{codes:} \mathrm{\# 1}-\mathrm{x}+1,-\mathrm{y}+1,-\mathrm{z}+1 ; \# 2 \mathrm{x}, \mathrm{y}, \mathrm{z}+1 ; \# 3-\mathrm{x}+1,-\mathrm{y}+1,-\mathrm{z} ; \# 4-\mathrm{x}+1,-\mathrm{y},-\mathrm{z} ; \# 5$ \\
$-\mathrm{x}+1,-\mathrm{y},-\mathrm{z}+1 ; \# 6-\mathrm{x}+2,-\mathrm{y}+1,-\mathrm{z}+1$. & & \\
\hline
\end{tabular}

Table S2 Average bond lengths of six-fold and seven-fold coordinated uranium cations in 1-8.

\begin{tabular}{lllll}
\hline & \multicolumn{2}{l}{ Six-fold coordinated $\mathrm{U}$ cations } & \multicolumn{2}{l}{ Seven-fold coordinated U cations } \\
& $\mathrm{U}=\mathrm{O}(\AA)$ & $\mathrm{U}-\mathrm{O}(\AA)$ & $\mathrm{U}=\mathrm{O}(\AA)$ & $\mathrm{U}-\mathrm{O}(\AA)$ \\
\hline $\mathbf{1}$ & 1.729 & 2.291 & 1.715 & 2.383 \\
$\mathbf{2}$ & -- & -- & 1.753 & 2.396 \\
$\mathbf{3}$ & -- & -- & 1.771 & 2.378 \\
$\mathbf{4}$ & 1.765 & 2.278 & 1.749 & 2.378 \\
$\mathbf{5}$ & 1.743 & 2.279 & 1.747 & 2.373 \\
$\mathbf{6}$ & 1.775 & 2.268 & 1.773 & 2.378 \\
$\mathbf{7}$ & 1.770 & 2.274 & 1.766 & 2.379 \\
$\mathbf{8}$ & 1.776 & 2.273 & 1.768 & 2.381 \\
\hline
\end{tabular}


Table S3 IR spectroscopic data for $\mathbf{1 - 8}$.

\begin{tabular}{|c|c|c|c|c|}
\hline & $\begin{array}{l}\mathrm{C}-\mathrm{H} \\
\text { (aromatic } \\
\text { ring) } / \mathrm{cm}^{-1}\end{array}$ & $\mathrm{C}=\mathrm{C}, \mathrm{C}-\mathrm{N}, \mathrm{COO} / \mathrm{cm}^{-1}$ & $\mathrm{O}=\mathrm{U}=\mathrm{O} / \mathrm{cm}^{-1}$ & $\mathrm{O}-\mathrm{P}-\mathrm{O}, \mathrm{P}-\mathrm{C} / \mathrm{cm}^{-1}$ \\
\hline 1 & & $\begin{array}{l}1563,1524,1434,1406 \\
1310\end{array}$ & $960,930,919,844$ & $1088,1041,742,695$ \\
\hline 2 & 3057 & $\begin{array}{l}1554,1526,1489,1432 \\
1404,1320\end{array}$ & $948,918,839$ & $1100,1030,750,690$ \\
\hline 3 & 3131 & $\begin{array}{l}1590,1519,1444,1404, \\
1312\end{array}$ & $948,909,856$ & $1100,1052,742,698$ \\
\hline 4 & 3131 & $\begin{array}{l}1584,1519,1440,1380, \\
1311\end{array}$ & $949,938,901,856$ & $1100,1011,723,698$ \\
\hline 5 & 3137 & $\begin{array}{l}1569,1525,1440,1405 \\
1310\end{array}$ & $932,903,839$ & $1057,1022,729,701$ \\
\hline 6 & 3136 & $\begin{array}{l}1590,1529,1435,1384, \\
1313\end{array}$ & $934,900,845$ & $1099,1016,743,691$ \\
\hline 7 & 3136 & $1590,1524,1435,1310$ & $936,904,841$ & $1100,1011,743,692$ \\
\hline 8 & & $1529,1454,1407,1313$ & 920,840 & $\begin{array}{l}1100,1042,770,733, \\
690\end{array}$ \\
\hline
\end{tabular}

Table S4 Comparison of selected single-crystal XRD and IR spectroscopic data for 1-8 using Veal's empirical law $R_{\mathrm{U}-\mathrm{O}}(\mathrm{pm})=8120 v_{3}^{-2 / 3}+89.5$.

\begin{tabular}{llll}
\hline & $v_{3}\left(\mathrm{~cm}^{-1}\right)$ in IR & Calculate from IR $(\mathrm{pm})$ & Single XRD data $(\mathrm{pm})$ \\
$\mathbf{1}$ & $960,929.7,919$ & $172.7,174.7,175.4$, & $169.5,172.9,173.3$ \\
$\mathbf{3}$ & 948,918 & $173.6,175.5$ & 175.3 \\
$\mathbf{4}$ & $948,909,856$ & $173.6,176.0,179.6$ & $176.3,177.7$ \\
$\mathbf{5}$ & $932,902.5$ & $173.6,174.2,176.5$ & $174.7,175.0,176.0,176.9$ \\
$\mathbf{6}$ & $934.3,900$ & $174.6,176.4$ & $174.2,174.3,174.4$ \\
$\mathbf{7}$ & $936.3,904$ & $174.5,176.6$ & $176.9,177.4,177.6$ \\
$\mathbf{8}$ & 919.7 & $174.3,176.4$ & $176.2,176.8,176.9$ \\
\hline
\end{tabular}


II. Powder X-ray diffraction patterns.

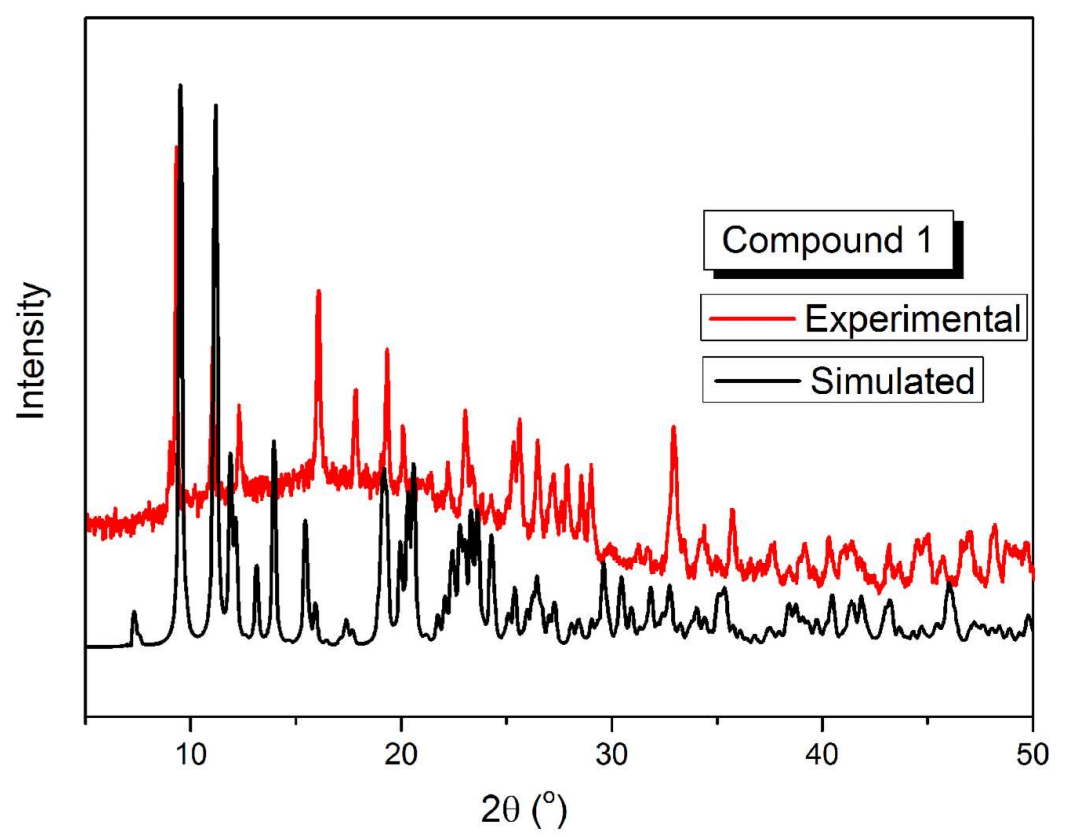

Figure S1. PXRD pattern of $\mathrm{Co}(\mathrm{im})_{2}\left(\mathrm{UO}_{2}\right)_{3}(\mathrm{~L})_{4} \mathbf{1}$.

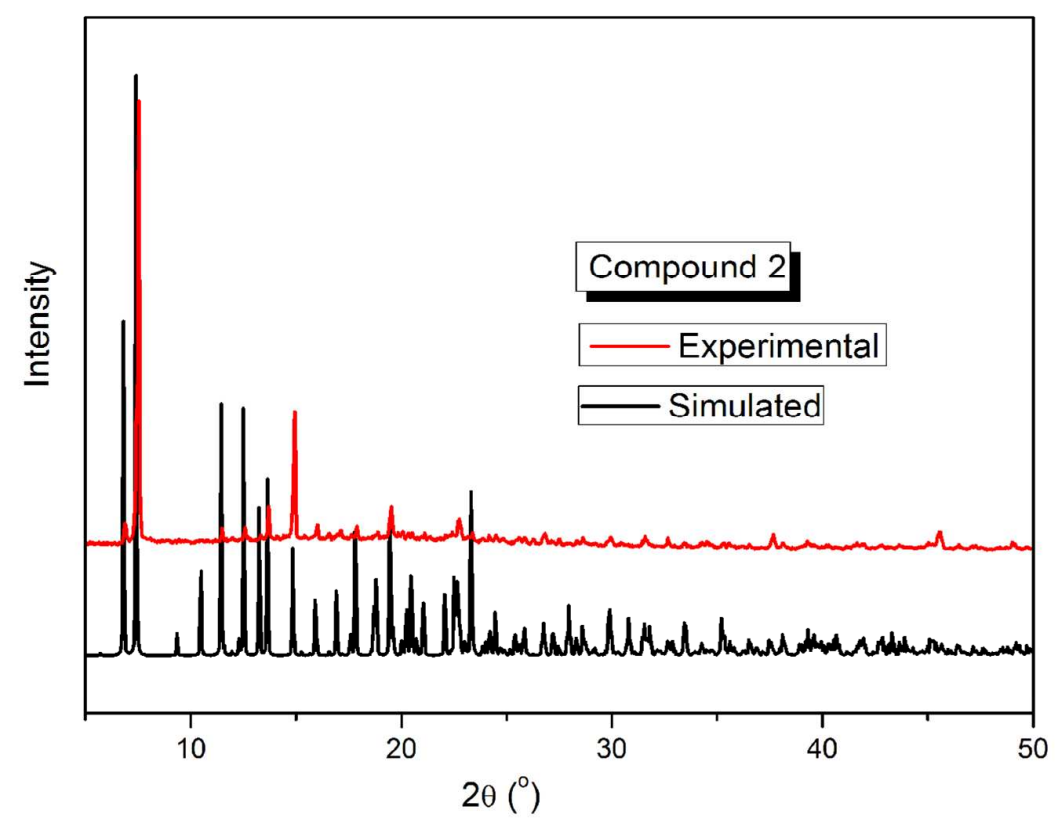

Figure S2. PXRD pattern of $\mathrm{Zn}(\mathrm{bpi})\left(\mathrm{UO}_{2}\right)(\mathrm{L})_{2} 2$. 


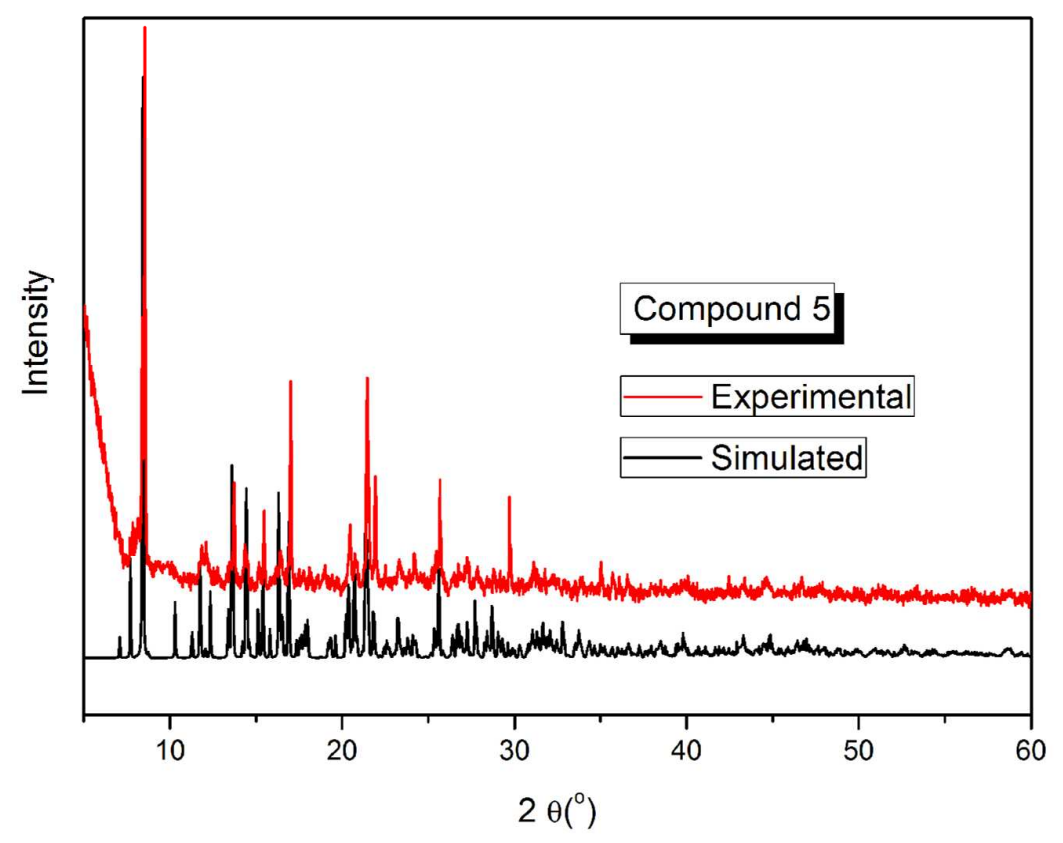

Figure S3. PXRD pattern of $\mathrm{Mn}(\mathrm{dib})\left(\mathrm{UO}_{2}\right)_{2}(\mathrm{~L})_{3} \mathbf{5}$.

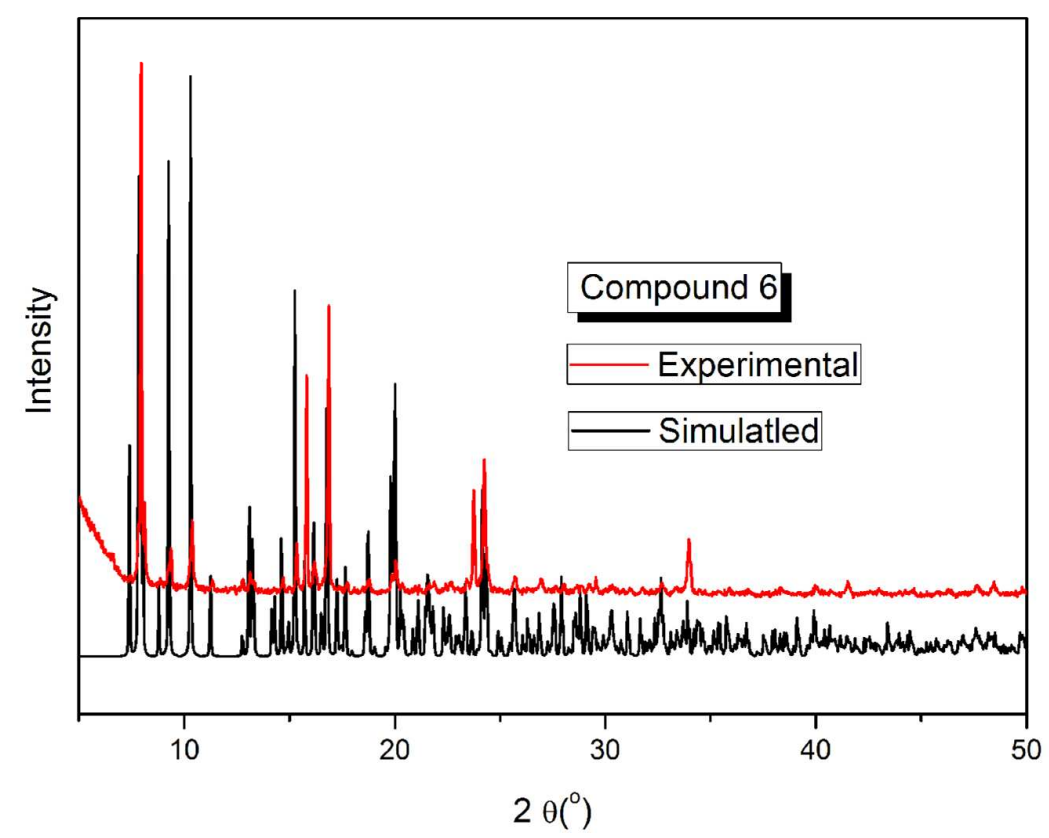

Figure S4. PXRD pattern of $\left[\mathrm{Co}(\mathrm{dib})_{2}\left(\mathrm{H}_{2} \mathrm{O}\right)_{2}\right]\left[\left(\mathrm{UO}_{2}\right)_{3}(\mathrm{~L})_{4}\right] \cdot 2 \mathrm{H}_{2} \mathrm{O} 6$. 


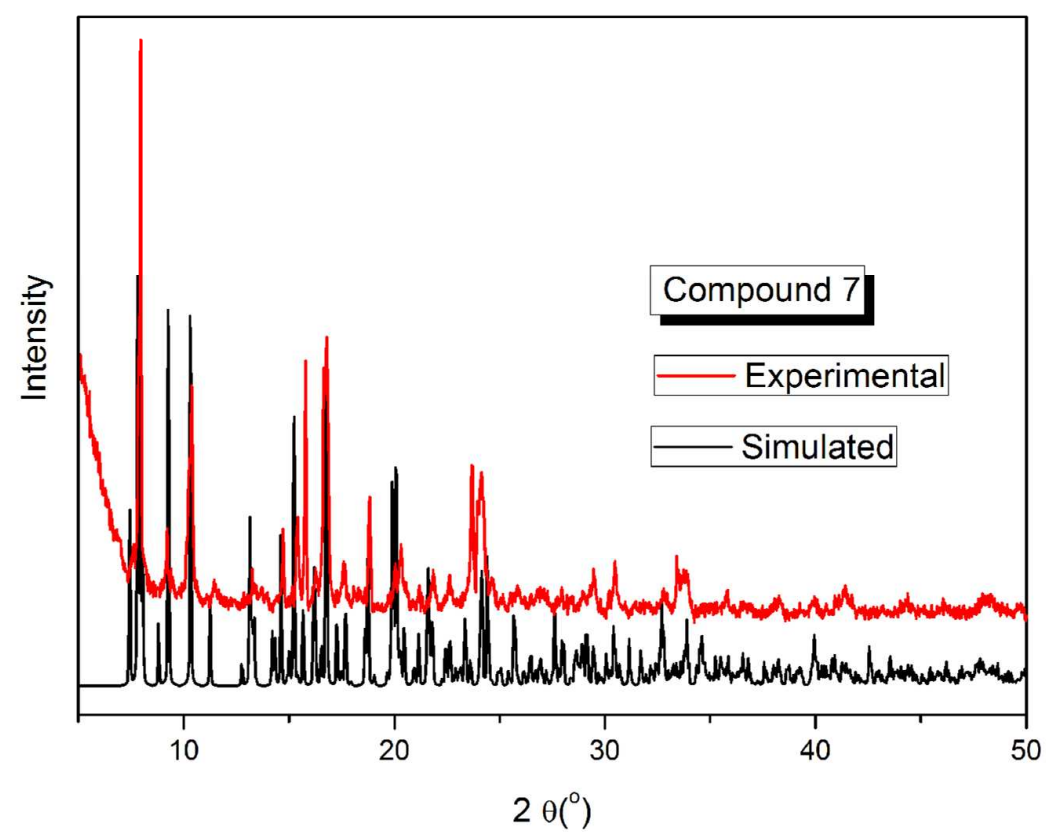

Figure S5. PXRD pattern of $\left[\mathrm{Ni}(\mathrm{dib})_{2}\left(\mathrm{H}_{2} \mathrm{O}\right)_{2}\right]\left[\left(\mathrm{UO}_{2}\right)_{3}(\mathrm{~L})_{4}\right] \cdot 2 \mathrm{H}_{2} \mathrm{O} 7$.

\section{Infrared spectra}

The strong peaks from 960 to $900 \mathrm{~cm}^{-1}$ are ascribed to the antisymmetric stretching modes $v_{3}$ of uranyl dication; whilst those in the range of $839-812 \mathrm{~cm}^{-1}$ are due to the symmetric stretching vibrations $v_{1}$. The bands in the range from 1100 to $1000 \mathrm{~cm}^{-1}$ and in the low wavenumber region from 750 to $690 \mathrm{~cm}^{-1}$ are dominated by the $\mathrm{O}-\mathrm{P}-\mathrm{O}$ bending and $\mathrm{P}-\mathrm{C}$ stretching vibrations. The vibrations of the aromatic rings are indicated in the area $1590-1310 \mathrm{~cm}^{-1}$. The peak located at $3130 \mathrm{~cm}^{-1}$ is attributed to the $\mathrm{C}-\mathrm{H}$ stretching vibrations of the aromatic ring. 


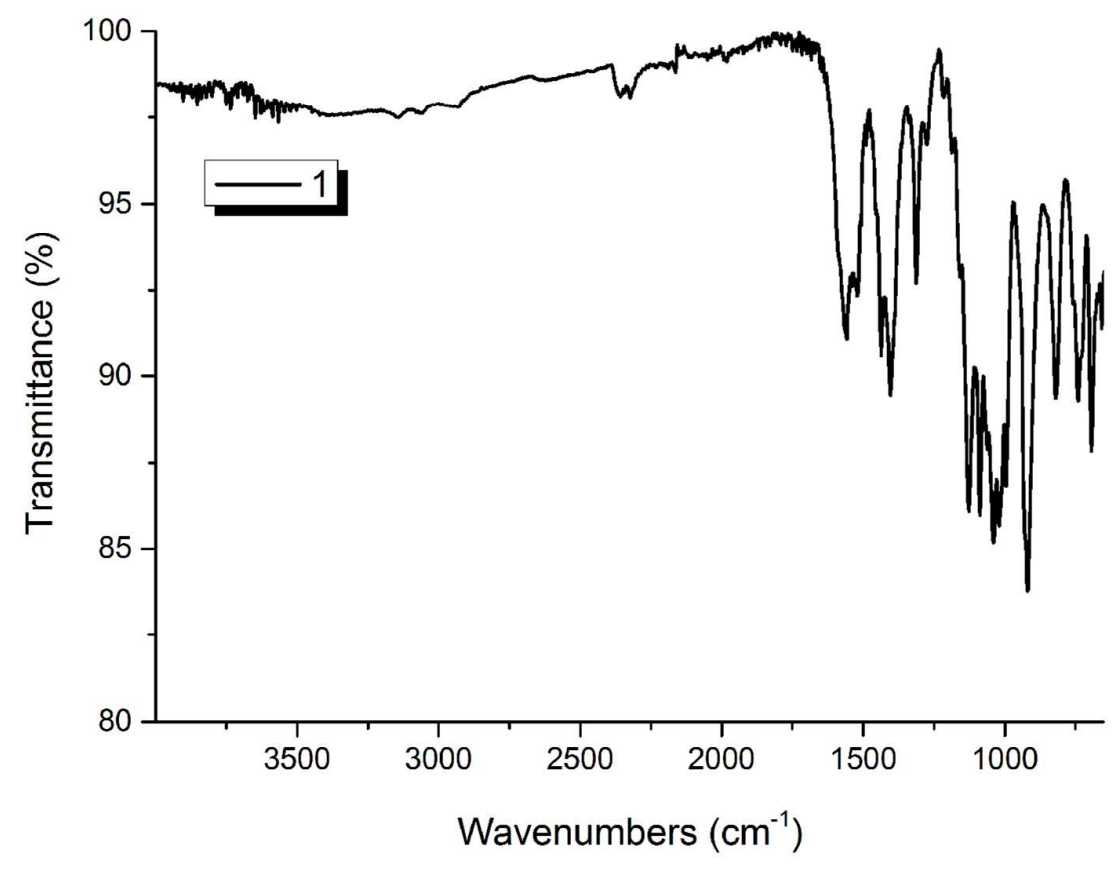

Figure S6. IR spectrum of $\mathrm{Co}(\mathrm{im})_{2}\left(\mathrm{UO}_{2}\right)_{3}(\mathrm{~L})_{4} \mathbf{1}$.

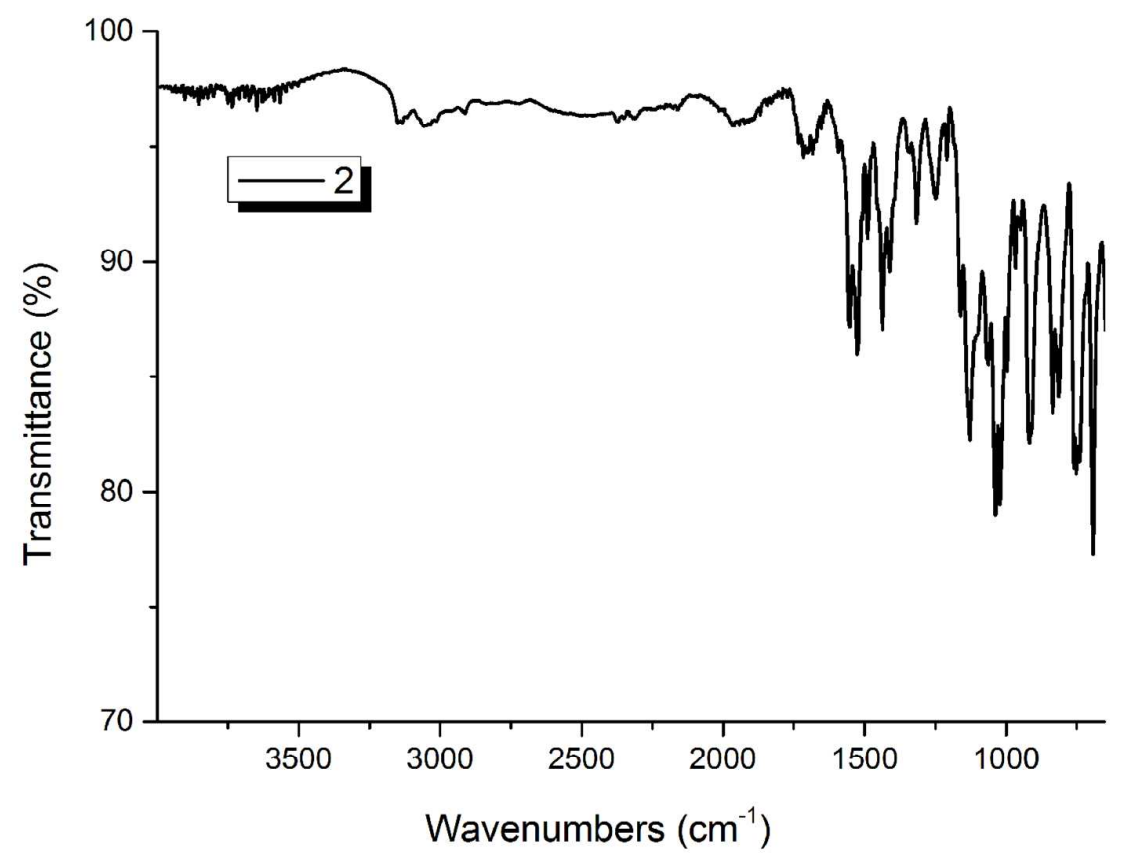

Figure S7. IR spectrum of $\mathrm{Zn}(\mathrm{bpi})\left(\mathrm{UO}_{2}\right)(\mathrm{L})_{2} 2$. 


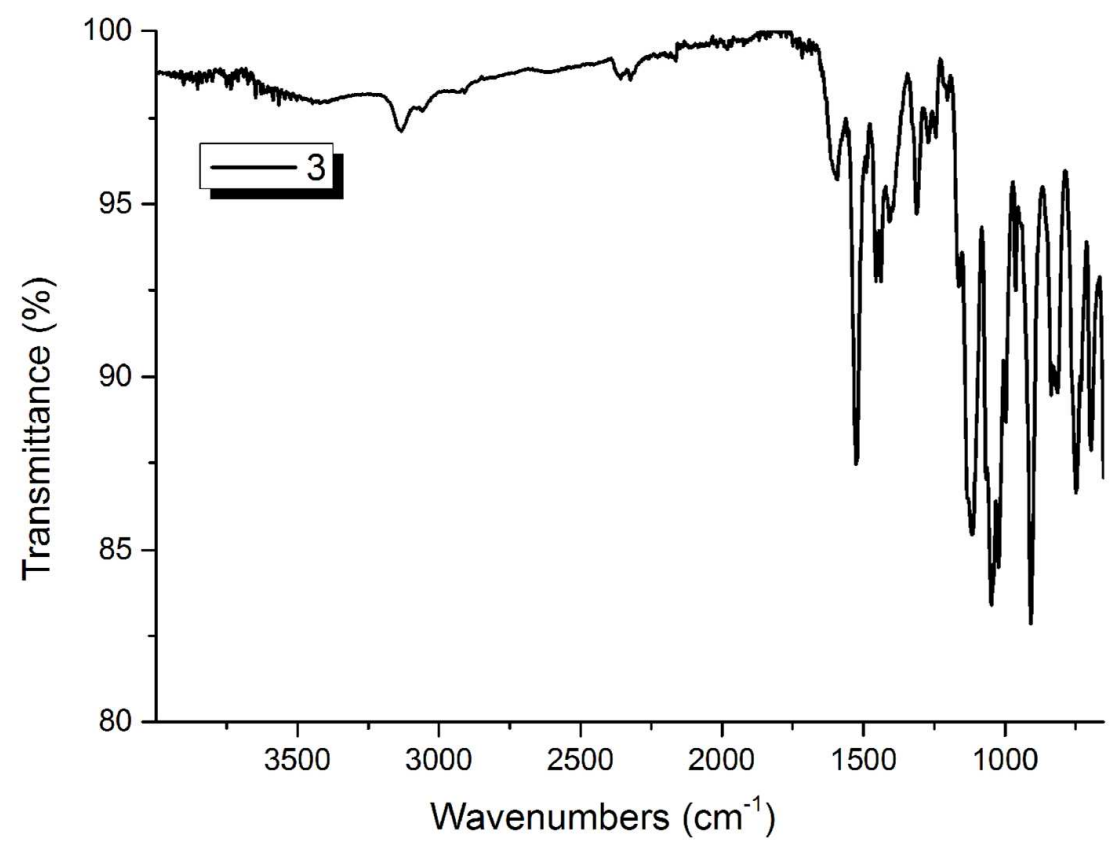

Figure S8. IR spectrum of $\mathrm{Cd}(\mathrm{dib})\left(\mathrm{UO}_{2}\right)(\mathrm{L})_{2} \mathbf{3}$.

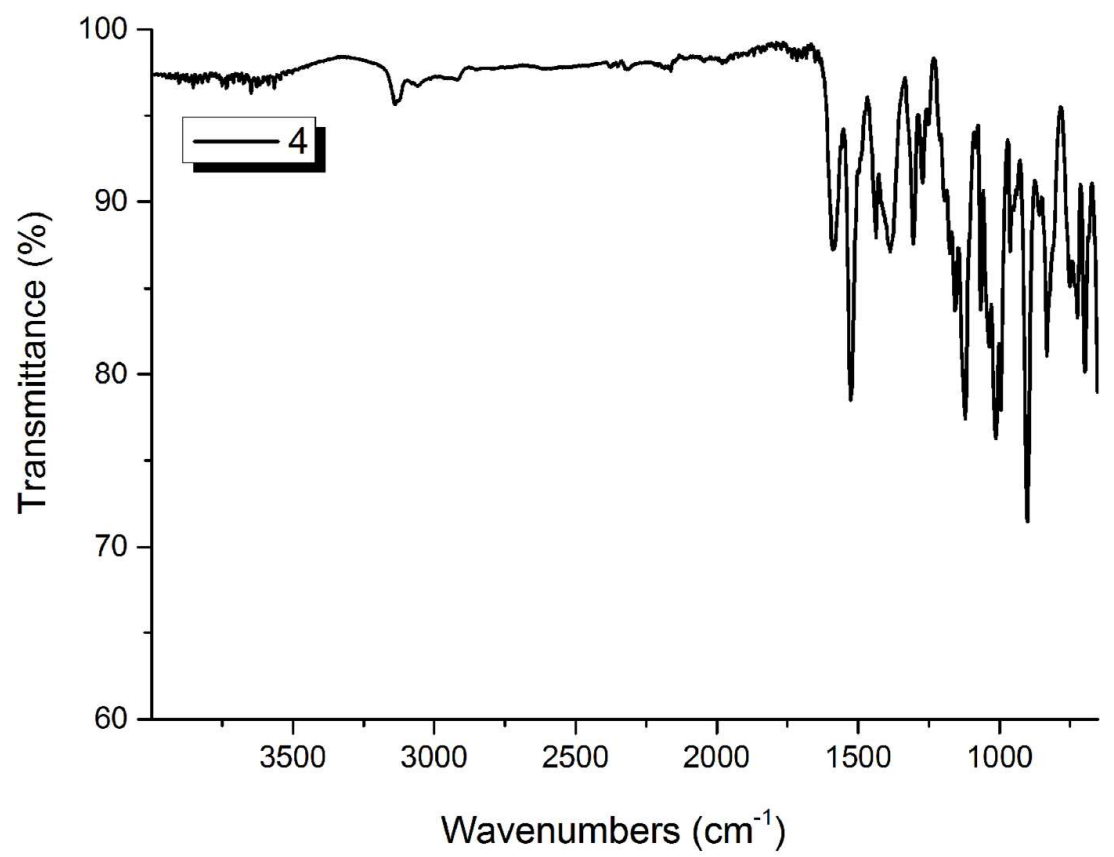

Figure S9. IR spectrum of $\mathrm{Cd}(\mathrm{dib})\left(\mathrm{UO}_{2}\right)_{2}(\mathrm{~L})_{3} 4$. 


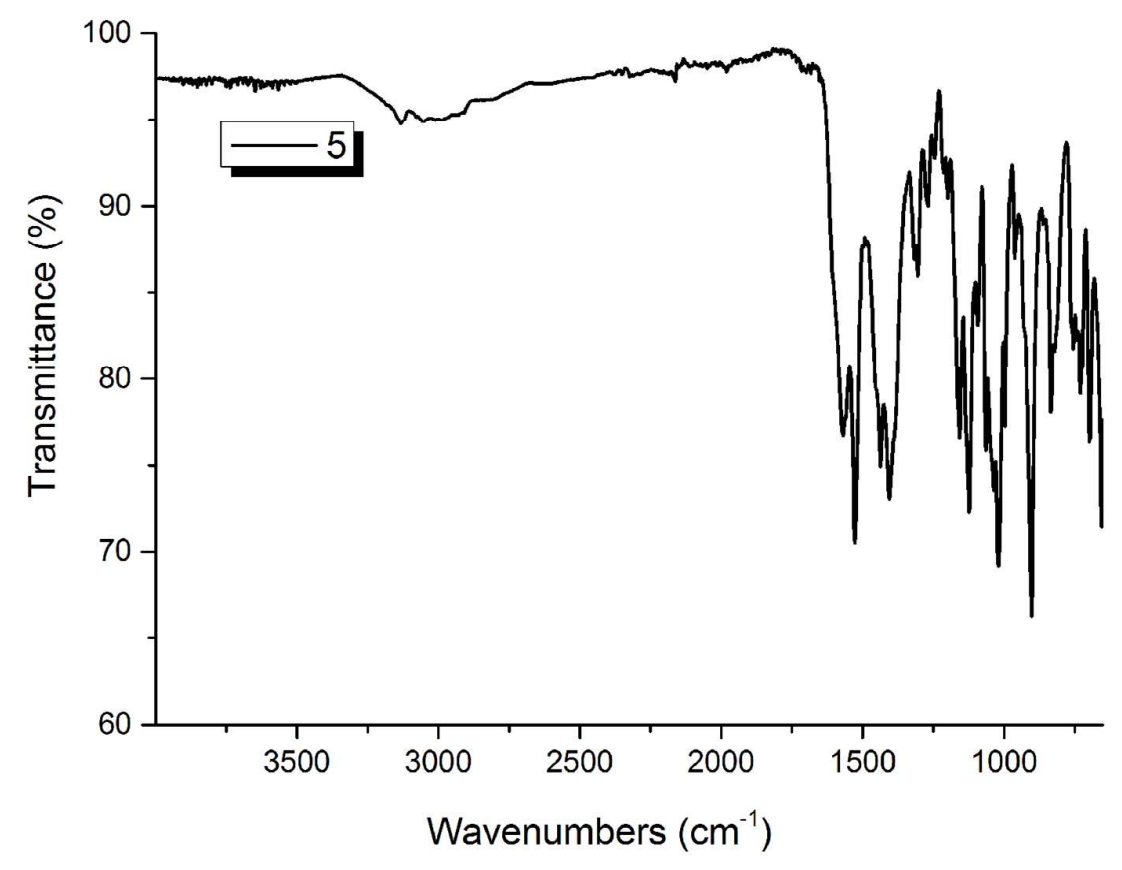

Figure S10. IR spectrum of $\mathrm{Mn}(\mathrm{dib})\left(\mathrm{UO}_{2}\right)_{2}(\mathrm{~L})_{3} \mathbf{5}$.

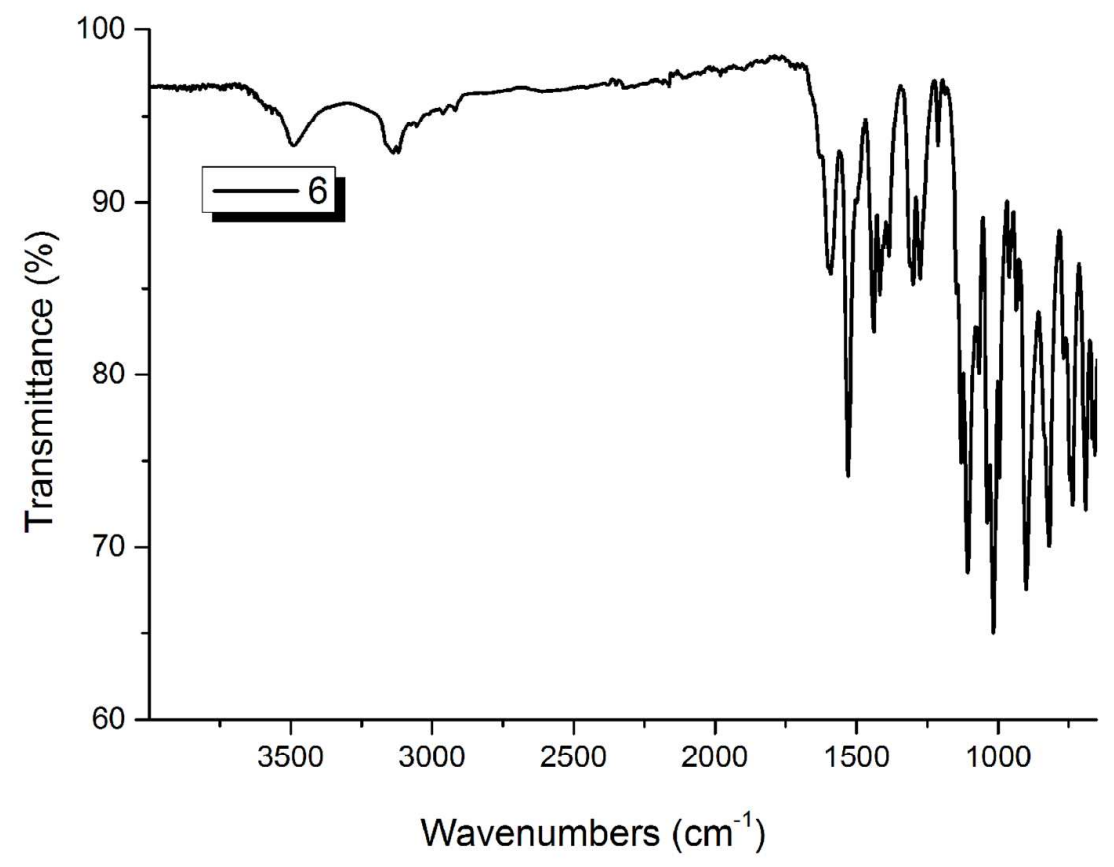

Figure $\mathrm{S} 11$. IR spectrum of $\left[\mathrm{Co}(\mathrm{dib})_{2}\left(\mathrm{H}_{2} \mathrm{O}\right)_{2}\right]\left[\left(\mathrm{UO}_{2}\right)_{3}(\mathrm{~L})_{4}\right] \cdot 2 \mathrm{H}_{2} \mathrm{O} 6$. 


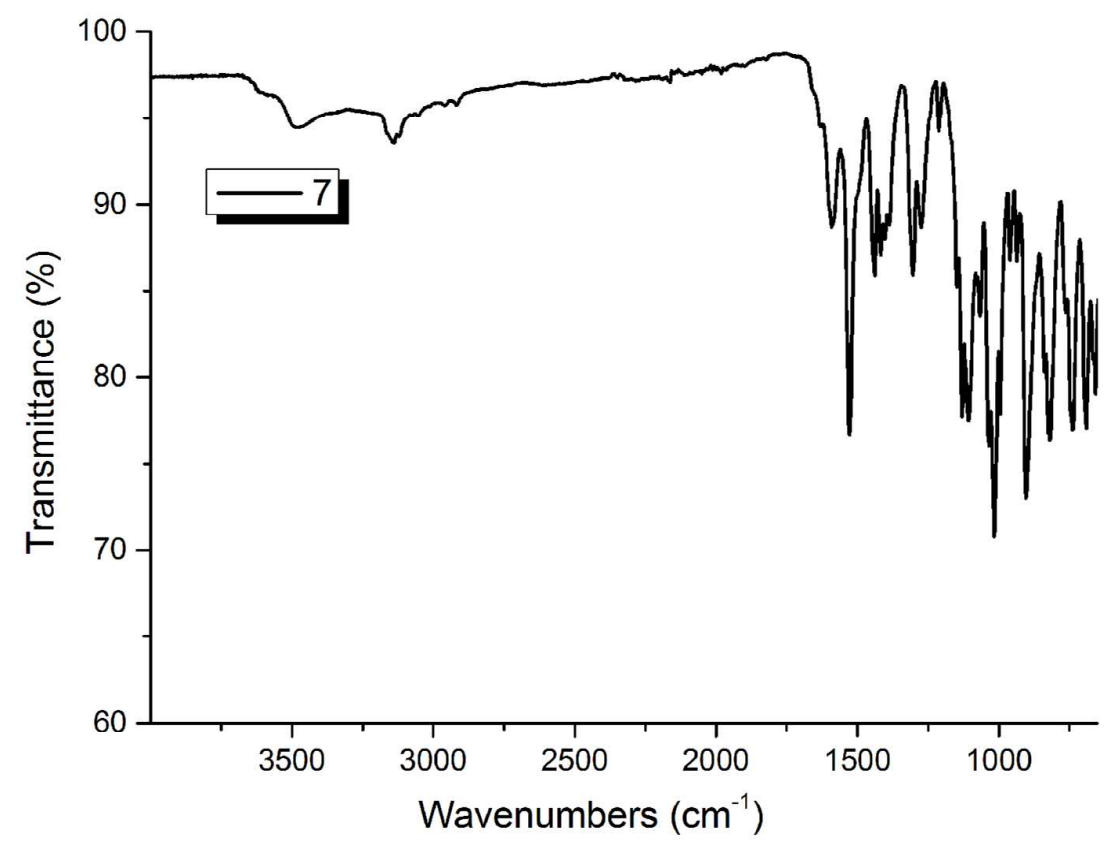

Figure S12. IR spectrum of $\left[\mathrm{Ni}(\mathrm{dib})_{2}\left(\mathrm{H}_{2} \mathrm{O}\right)_{2}\right]\left[\left(\mathrm{UO}_{2}\right)_{3}(\mathrm{~L})_{4}\right] \cdot 2 \mathrm{H}_{2} \mathrm{O} 7$.

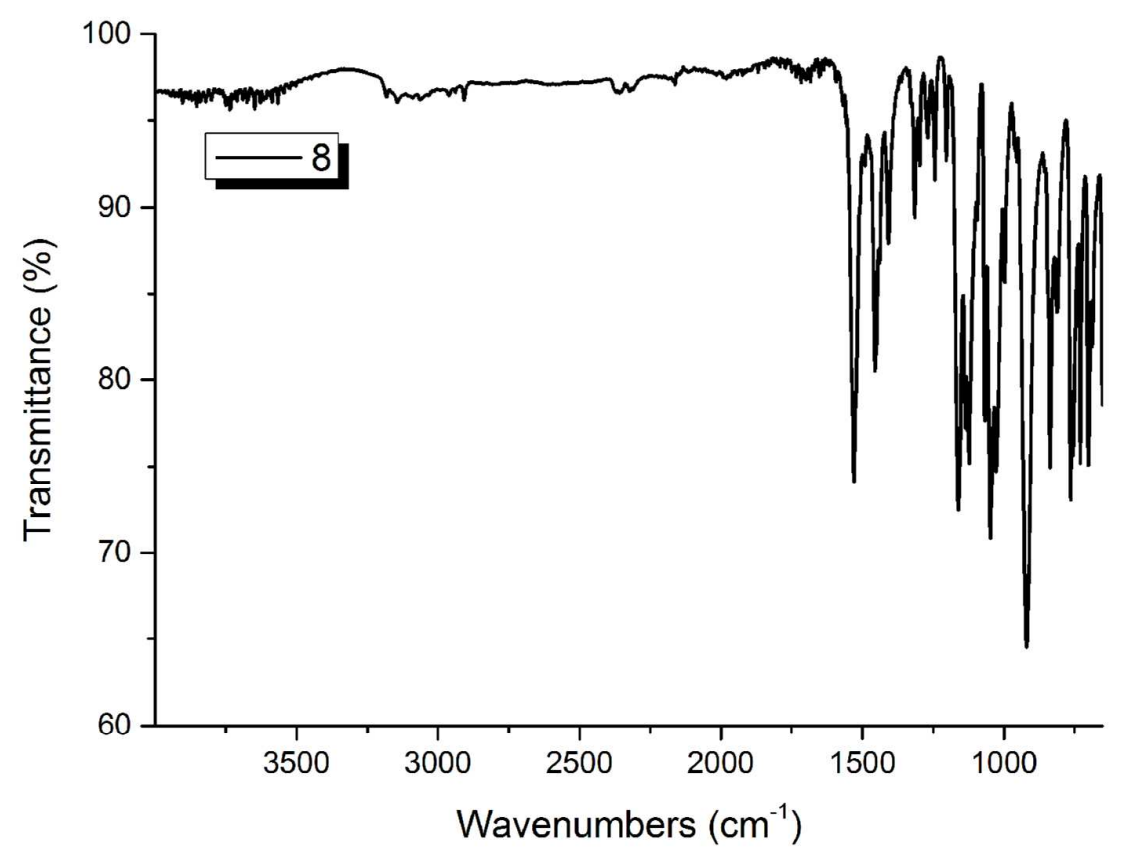

Figure S13. IR spectrum of $\left[\mathrm{Cu}(\mathrm{dib})_{2}\left(\mathrm{H}_{2} \mathrm{O}\right)_{2}\right]\left[\left(\mathrm{UO}_{2}\right)_{3}(\mathrm{~L})_{4}\right] 8$.

IV. ORTEP representation of local structures. 


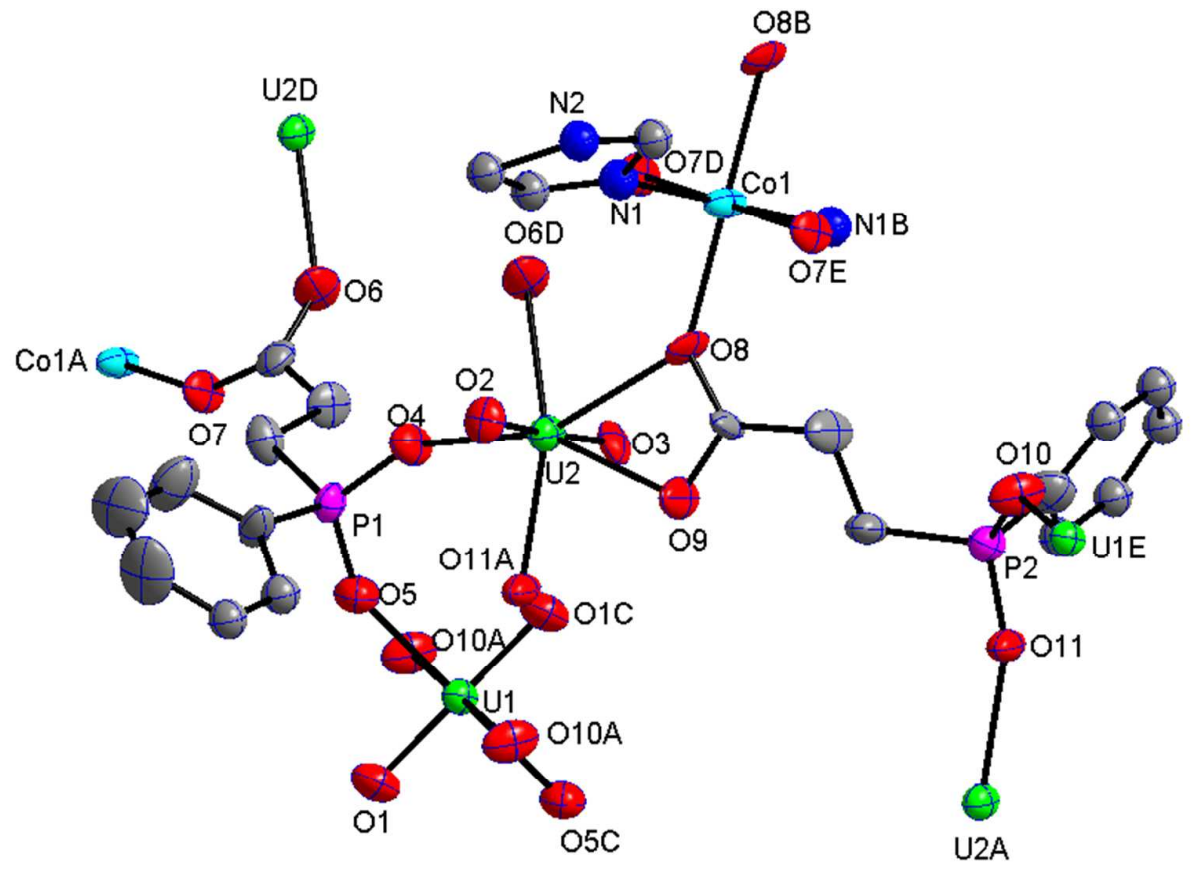

Figure S14. ORTEP representation of local structure of $\mathrm{Co}(\mathrm{im})_{2}\left(\mathrm{UO}_{2}\right)_{3}(\mathrm{~L})_{4} \mathbf{1}$. Symmetry codes: A, 2-x, -y, 1-z; B, 2-x, 1-y, 1-z; C, 2-x, -y, -z; D, 2-x, 1-y, -z; E, x, y, $1+\mathrm{z}$.

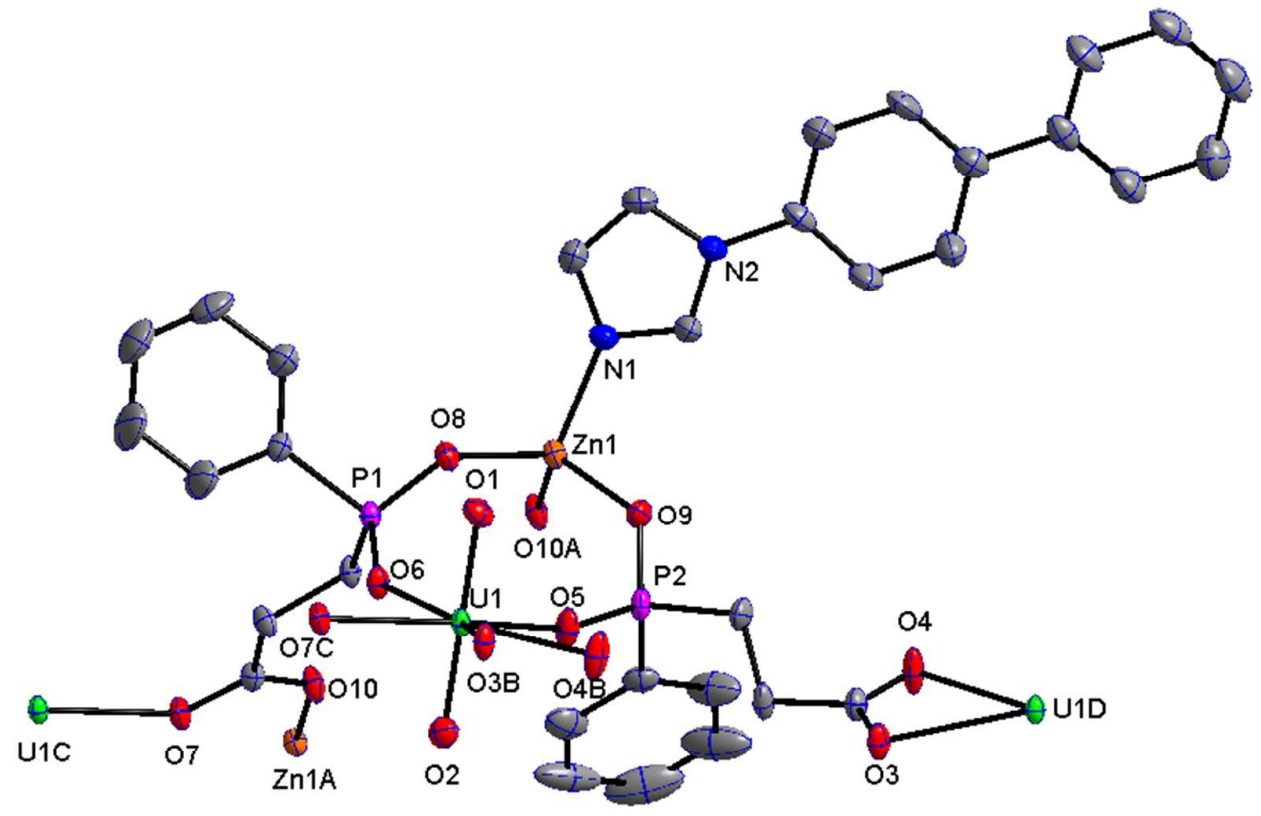

Figure S15. ORTEP representation of local structure of $\mathrm{Zn}(\mathrm{bpi})\left(\mathrm{UO}_{2}\right)(\mathrm{L})_{2} \boldsymbol{2}$. Symmetry codes: A, 1-x, 2-y, 1-z; B, 1-x, 0.5+y, 1.5-z; C, 1-x, 3-y, 1-z; D, 1-x, -0.5+y, $1.5-\mathrm{z}$. 


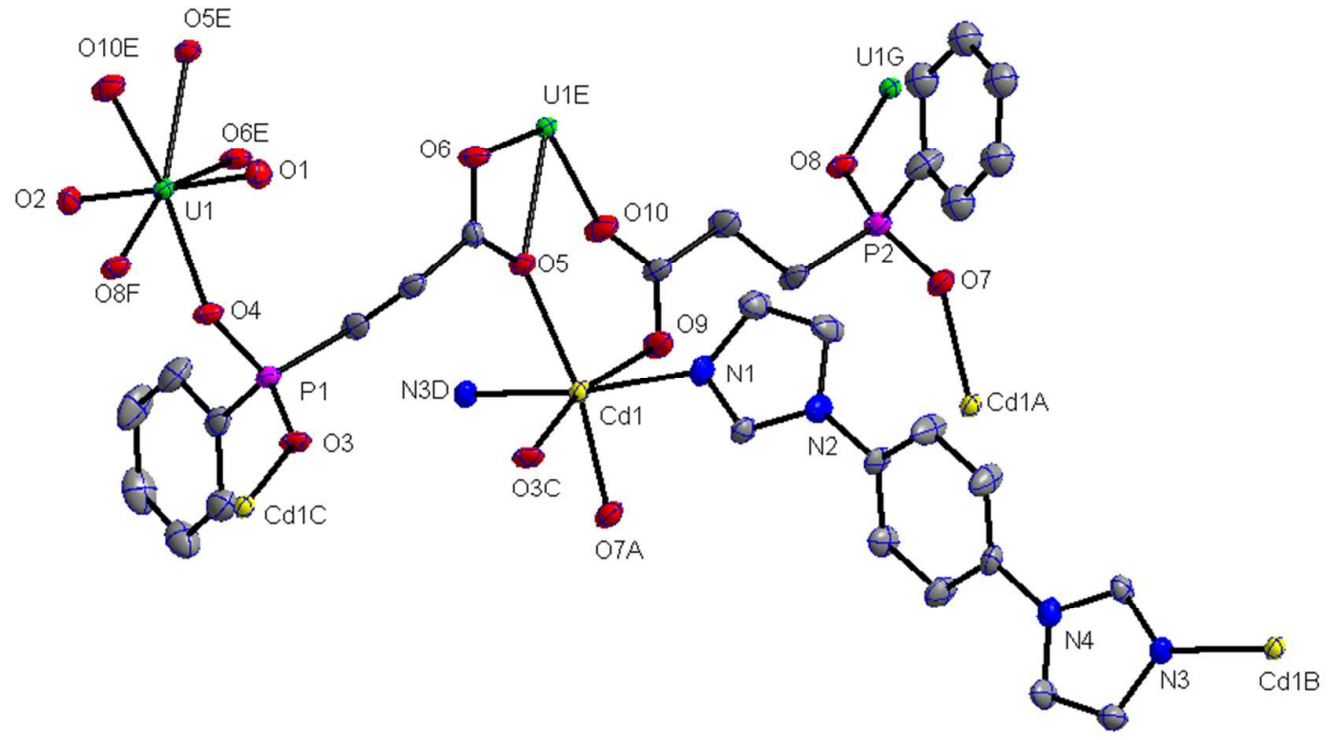

Figure S16. ORTEP representation of local structure of $\mathrm{Cd}(\mathrm{dib})\left(\mathrm{UO}_{2}\right)(\mathrm{L})_{2} 3$. Symmetry codes: A, -x, -y, 2-z; B, -1+x, 1+y, z; C, -x, -y, 1-z; D, 1+x, -1+y, z; E, 1-x, $-\mathrm{y}, 1-\mathrm{z} ; \mathrm{F}, \mathrm{x}, \mathrm{y},-1+\mathrm{z} ; \mathrm{G}, \mathrm{x}, \mathrm{y}, 1+\mathrm{z}$.

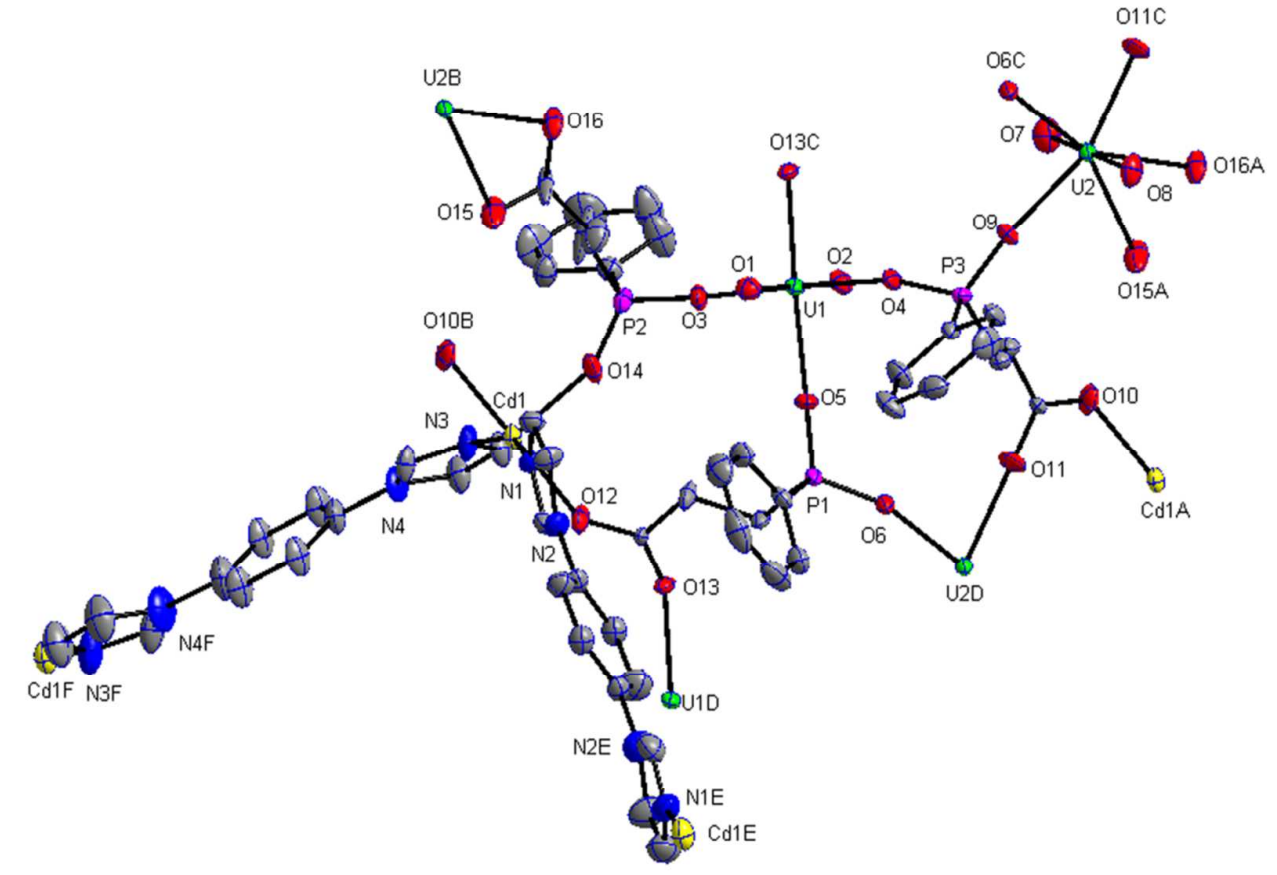

Figure S17. ORTEP representation of local structure of $\mathrm{Cd}(\mathrm{dib})\left(\mathrm{UO}_{2}\right)_{2}(\mathrm{~L})_{3} 4$. Symmetry codes: A, x, 1+y, z; B, x, -1+y, z; C, -1+x, y, z; D, 1+x, y, z; E, 2-x, -y, 1-z; F, 2-x, -1-y, -z. 


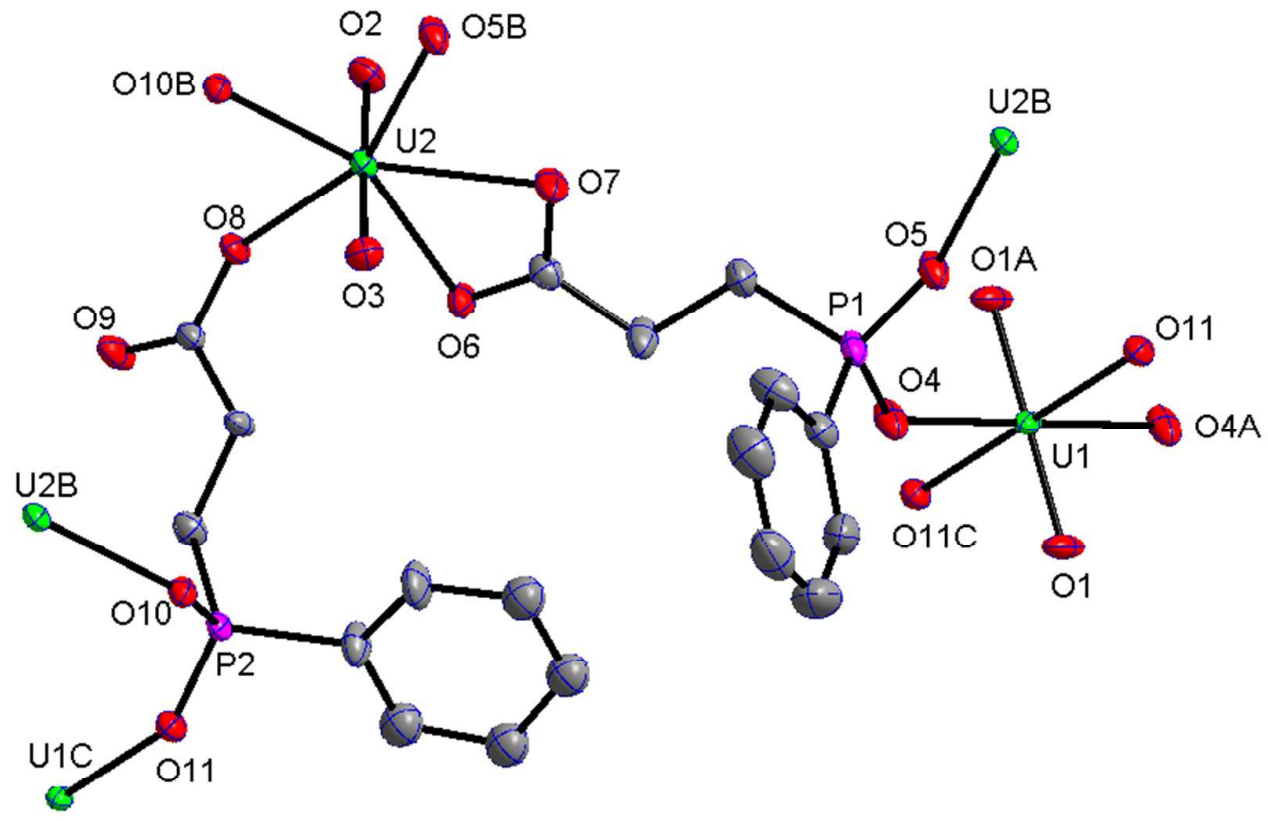

Figure S18. ORTEP representation of local structure of $\left[\mathrm{Co}(\mathrm{dib})_{2}\left(\mathrm{H}_{2} \mathrm{O}\right)_{2}\right]\left[\left(\mathrm{UO}_{2}\right)_{3}(\mathrm{~L})_{4}\right] \cdot 2 \mathrm{H}_{2} \mathrm{O}$ 6. Symmetry codes: A, 1-x, 1-y, 1-z; B, 1-x, -y, $1-z ; C, x, y,-1+z$.

V. Excitation and Emission spectra.

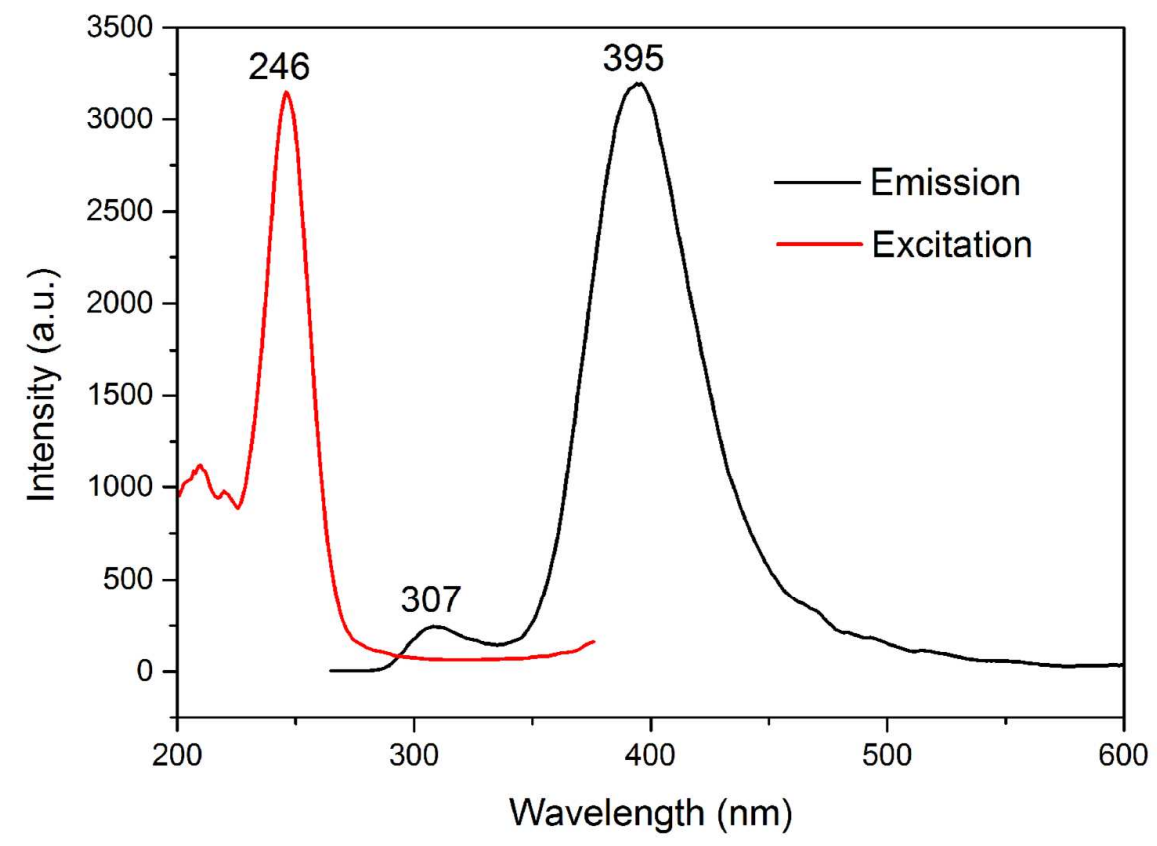

Figure S19. Excitation and emission spectra of Co(im $)_{2}\left(\mathrm{UO}_{2}\right)_{3}(\mathrm{~L})_{4} \mathbf{1}$. 


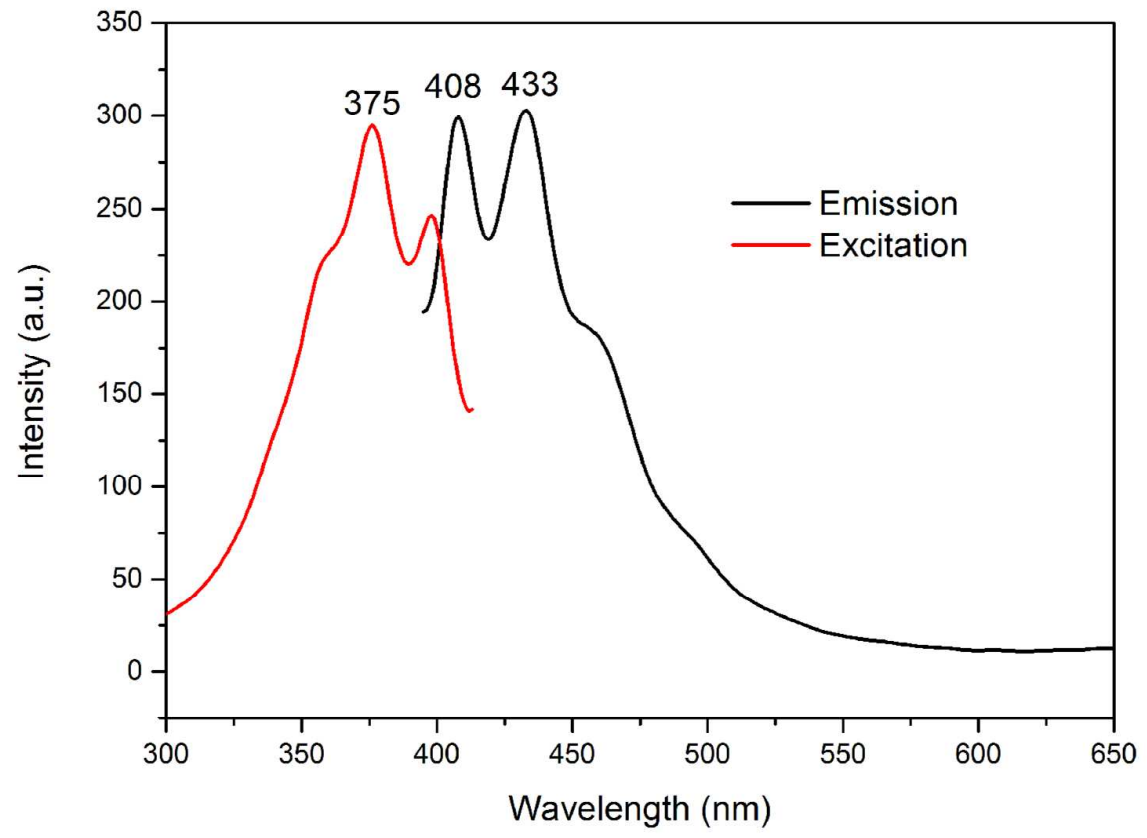

Figure S20. Excitation and emission spectra of $\mathrm{Mn}(\mathrm{dib})\left(\mathrm{UO}_{2}\right)_{2}(\mathrm{~L})_{3} 5$.

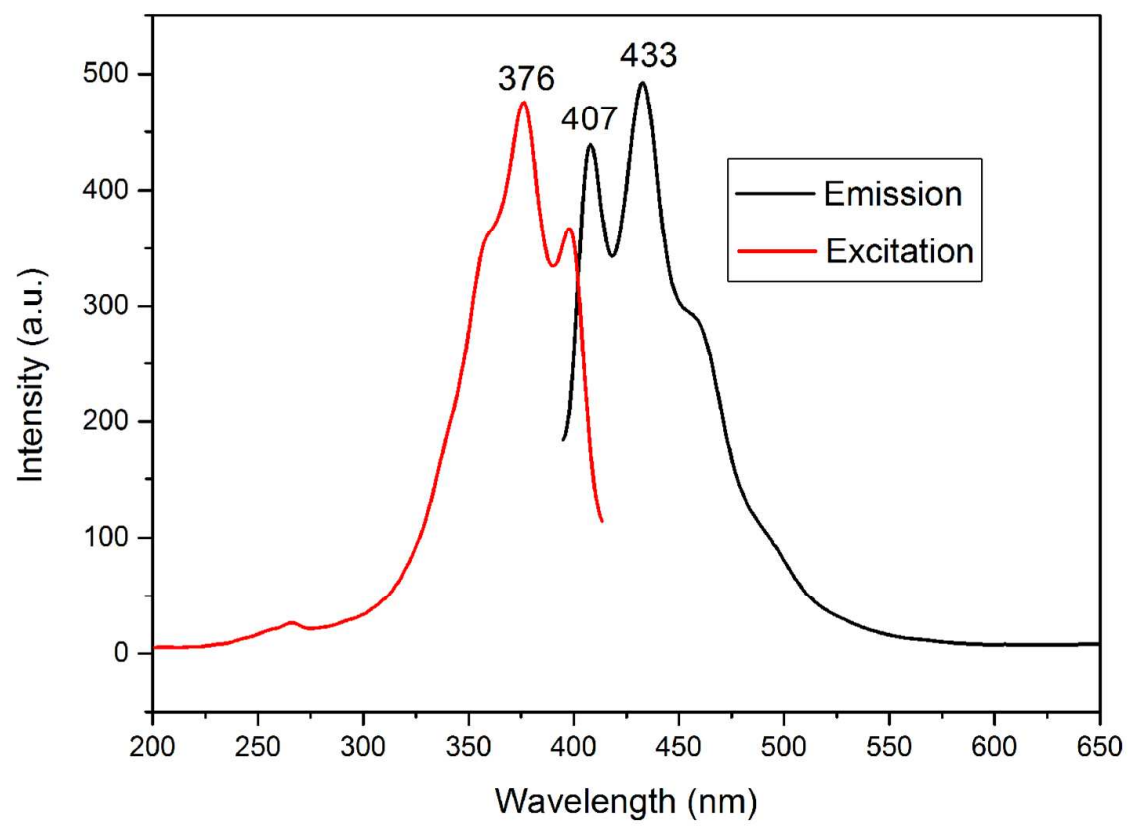

Figure S21. Excitation and emission spectra of $\left[\mathrm{Co}(\mathrm{dib})_{2}\left(\mathrm{H}_{2} \mathrm{O}\right)_{2}\right]\left[\left(\mathrm{UO}_{2}\right)_{3}(\mathrm{~L})_{4}\right] \cdot 2 \mathrm{H}_{2} \mathrm{O}$ 6. 


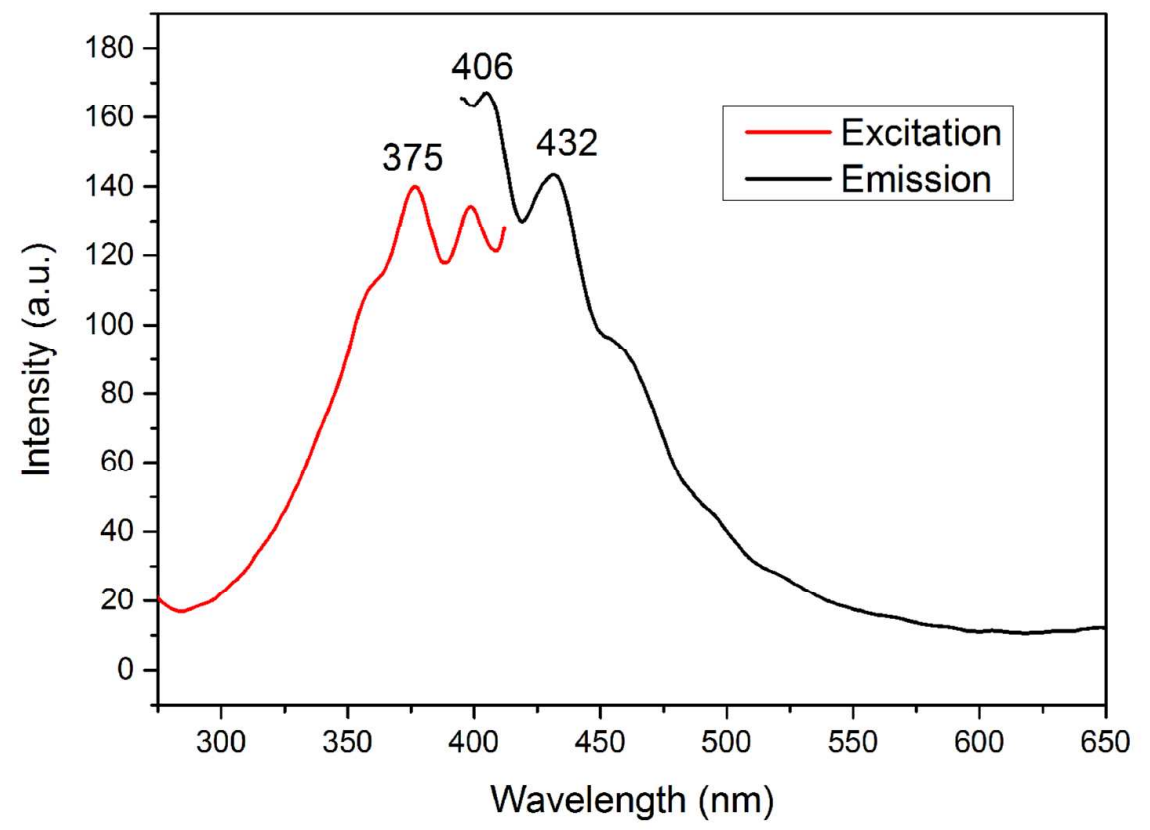

Figure S22. Excitation and emission spectra of $\left[\mathrm{Ni}(\mathrm{dib})_{2}\left(\mathrm{H}_{2} \mathrm{O}\right)_{2}\right]\left[\left(\mathrm{UO}_{2}\right)_{3}(\mathrm{~L})_{4}\right] \cdot 2 \mathrm{H}_{2} \mathrm{O}$ 7.

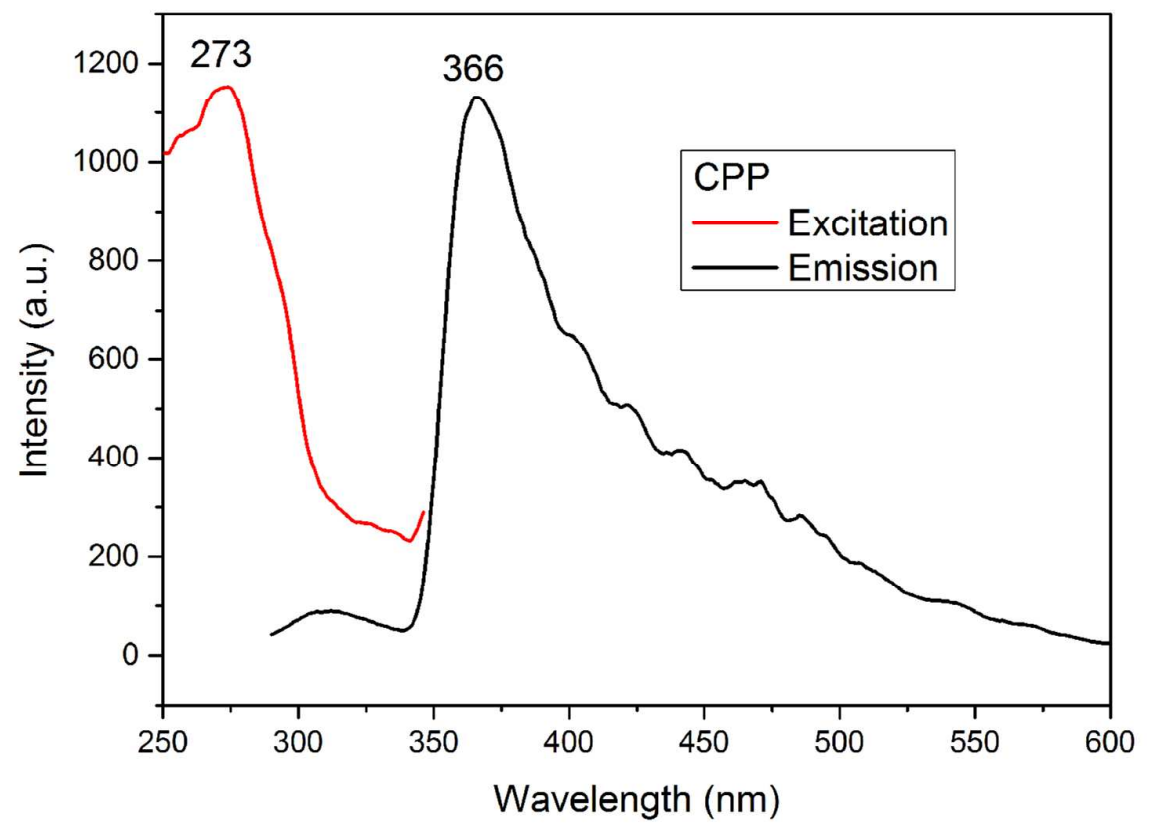


Figure S23. Excitation and emission spectra of the carboxphosphinate ligand CPP.

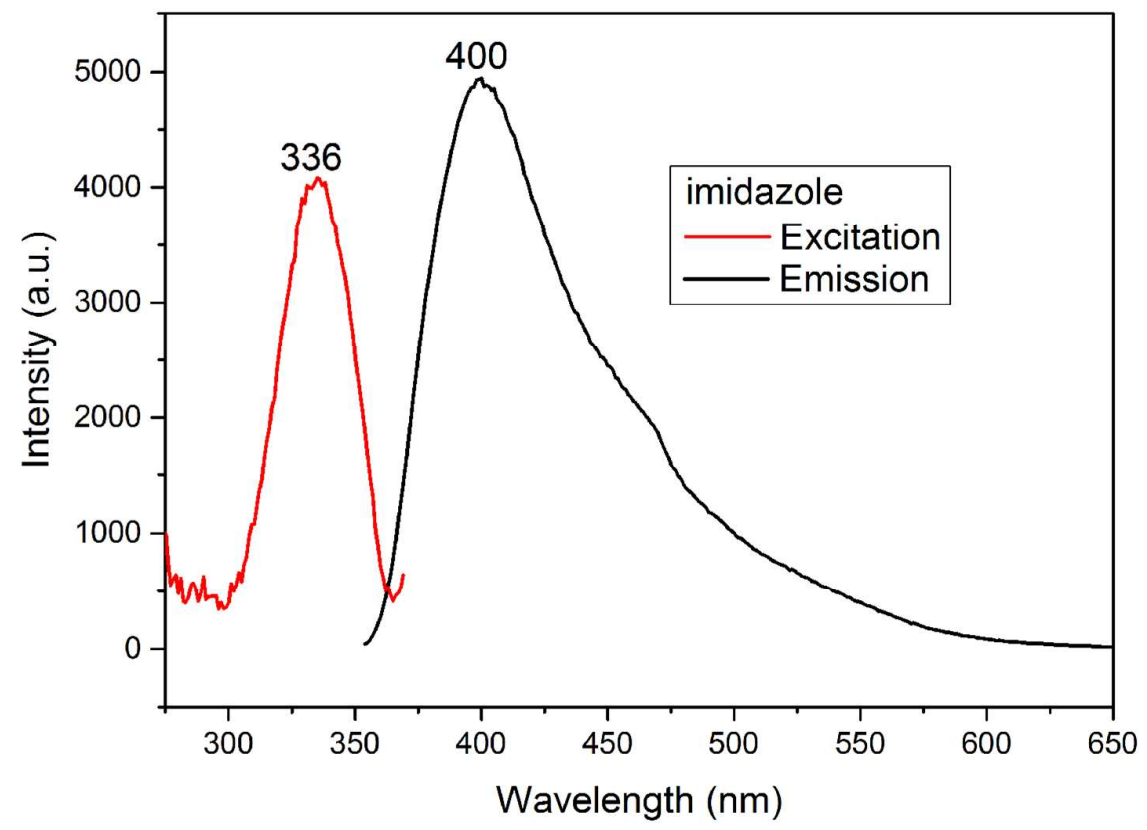

Figure S24. Excitation and emission spectra of imidazole.

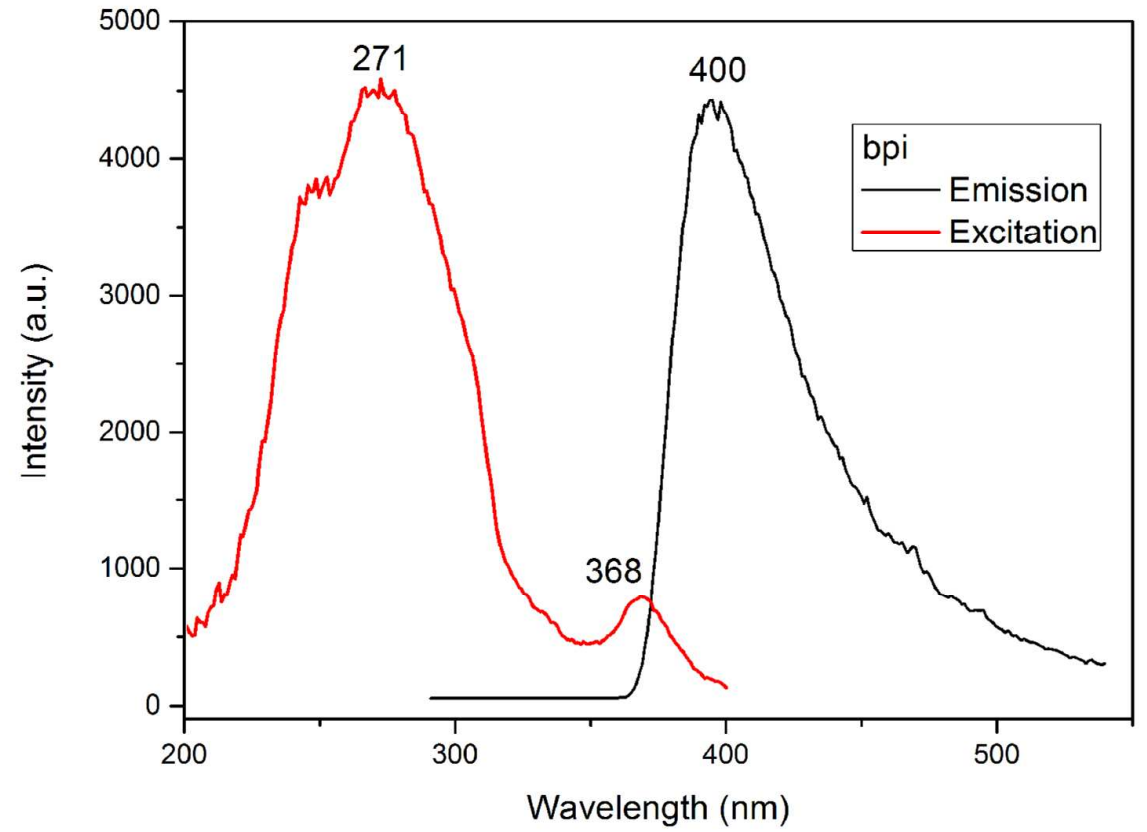

Figure S25. Excitation and emission spectra of bpi. 


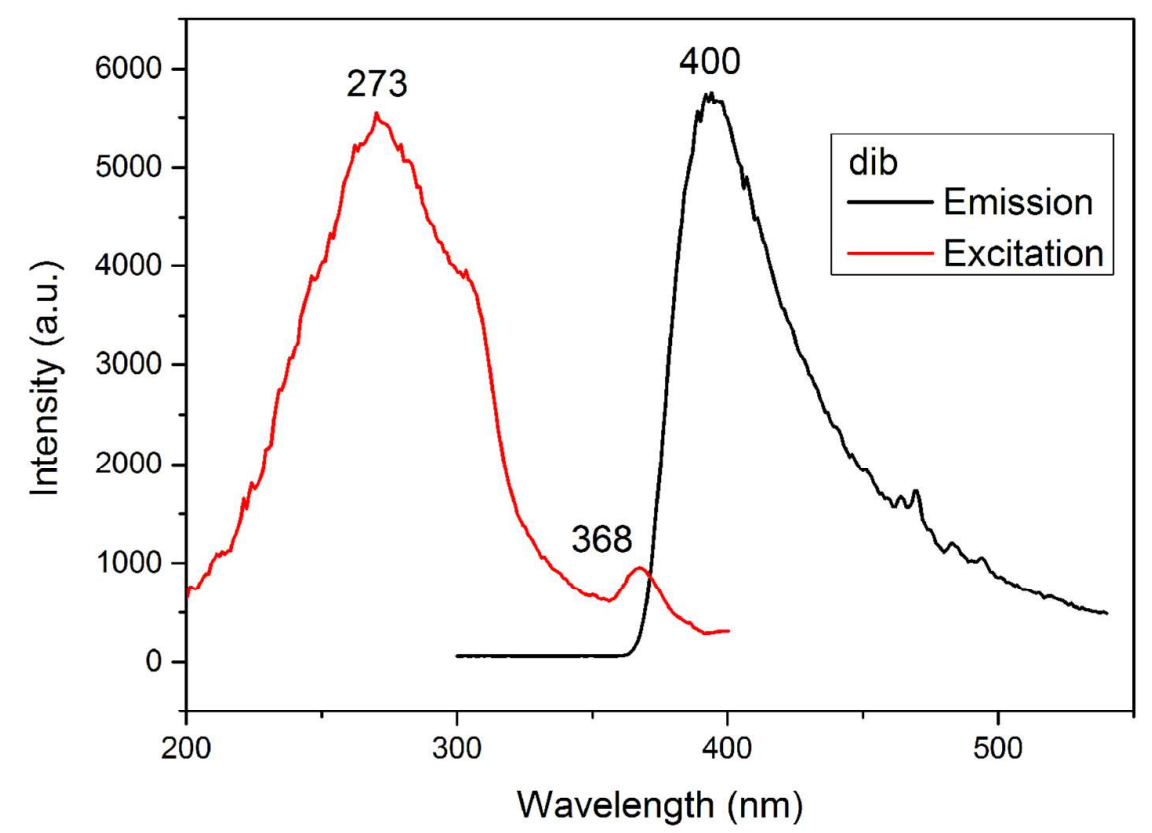

Figure S26. Excitation and emission spectra of dib. 